\title{
Consensus and Disagreement of Heterogeneous Belief Systems in Influence Networks
}

\author{
Mengbin Ye, Member, IEEE Ji Liu, Member, IEEE Lili Wang, Student Member, IEEE \\ Brian D.O. Anderson, Life Fellow, IEEE Ming Cao, Senior Member, IEEE
}

\begin{abstract}
Recently, an opinion dynamics model has been proposed to describe a network of individuals discussing a set of logically interdependent topics. For each individual, the set of topics and the logical interdependencies between the topics (captured by a logic matrix) form a belief system. We investigate the role the logic matrix and its structure plays in determining the final opinions, including existence of the limiting opinions, of a strongly connected network of individuals. We provide a set of results that, given a set of individuals' belief systems, allow a systematic determination of which topics will reach a consensus, and which topics will disagreement in arise. For irreducible logic matrices, each topic reaches a consensus. For reducible logic matrices, which indicates a cascade interdependence relationship, conditions are given on whether a topic will reach a consensus or not. It turns out that heterogeneity among the individuals' logic matrices, and a cascade interdependence relationship, are necessary conditions for disagreement. This paper thus attributes, for the first time, a strong diversity of limiting opinions to heterogeneity of belief systems in influence networks, in addition to the more typical explanation that strong diversity arises from individual stubbornness.
\end{abstract}

Index Terms-opinion dynamics, social networks, multi-agent systems, influence networks, agent-based models

\section{INTRODUCTION}

$\mathbf{T}$ HERE has been great interest over the past few years in agent-based network models of opinion dynamics that describe how individuals' opinions on a topic evolve over time as they interact [1], [2]. The seminal discrete-time French-Harary-DeGroot model [3]-[5] (or DeGroot model for short) assumes that each individual's opinion at the next time step is a convex combination of his/her current opinion and the current opinions of his/her neighbours. This weighted averaging aims to capture social influence, where individuals exert a conforming influence on each other so that over time, opinions become more similar (and thus giving rise to the term "influence network"). For networks satisfying mild

M. Ye and M. Cao are supported in part by the European Research Council (ERC-CoG-771687) and the Netherlands Organization for Scientific Research (NWO-vidi-14134). B. D.O. Anderson is supported by the Australian Research Council (ARC) under grant DP160104500 and DP190100887, and by Data61CSIRO.

M. Ye and M. Cao are with the Faculty of Science and Engineering, ENTEG, University of Groningen, Groningen 9747 AG, Netherlands.

M. Ye and B.D.O. Anderson are with the Research School of Engineering, Australian National University, Canberra, Australia. B.D.O. Anderson is also with Hangzhou Dianzi University, China, and with Data61-CSIRO in Canberra, Australia.

J. Liu is with the Department of Electrical and Computer Engineering, Stony Brook University.

L. Wang is with the Department of Electrical Engineering, Yale University.

$\{\mathrm{m}$.ye,m.cao\}@rug.nl, brian.anderson@anu.edu.au,

ji.liuestonybrook.edu, lili.wang@yale.edu. connectivity conditions, the opinions reach a consensus, i.e. the opinion values are equal for all individuals.

Since then, and to reflect real-world networks, much focus has been placed on developing models of increasing sophistication to capture different socio-psychological features that may be involved when individuals interact. The HegselmannKrause model [6]-[8] introduced the concept of bounded confidence, which is used to capture homophily, i.e. the phenomenon whereby individuals only interact with those other individuals whose opinion values are similar to their own. The Altafini model [9]-[12] introduced negative edge weights to model antagonistic or competitive interactions between individuals (perhaps arising from mistrust). The FriedkinJohnsen model generalised the DeGroot model by introducing the idea of "stubbornness", where an individual remains (at least partially) attached to his or her initial opinion [13], [14]. Of particular note is that the DeGroot and Friedkin-Johnsen models have been empirically examined [14]-[16]. For more detailed discussions on opinion dynamics modelling, we refer the reader to [1], [2], [17].

Recently in [18], a multi-dimensional extension to the Friedkin-Johnsen model was proposed to describe a network of individuals who simultaneously discuss a set of logically interdependent topics. That is, an individual's position on Topic $A$ may influence his/her position on Topic $B$ due to his/her view of constraints or relations between the two topics. Such interdependencies are captured in the model by a "logic matrix". This interdependence can greatly shift the final opinion values on the set of topics since now the interdependencies and the social influence from other individuals both affect opinion values. The model is used in [19] to explain that the shift in the US public's opinions on the topic of whether the 2003 Invasion of Iraq was justified was due to shifting opinions on the logically interdependent topic of whether Iraq had weapons of mass destruction. The set of topics, the interdependent functionalities between the topics, and the mechanism by which an individual processes such interdependencies forms a "belief system" as termed by Converse in his now classical paper [20]. For networks where all individuals have the same logic matrix, a complete stability result is given using algebraic conditions in [18] and using graph-theoretic conditions in [21]. Of course, the assumption that all individuals have the same logic matrix is restrictive. Heterogeneous logic matrices were considered in [19], but at least one individual is required to exhibit stubbornness in order to obtain a stability result.

This paper will also consider a generalisation of the multi- 
dimensional model proposed in [18] for the evolution of opinions in belief systems, going beyond [18], [19] by analysing the effects of the logic matrix, including especially heterogeneity of the logic matrices among the individuals, on the limiting opinion distribution. We first establish a general convergence result for the model with heterogeneous logic matrices on strongly connected networks. Then, we provide a set of results which enables the systematic determination of whether for a given topic, the opinions of the individuals will reach a consensus, or will reach a state of persistent disagreement.

We find that the nature of the logic structure, viz. the heterogeneity among the individuals of the logical interdependencies between topics, and the structure itself, plays a major role in determining whether opinions on a given topic reach a consensus or fail to do so. If the logical interdependencies do not have a cascade structure, then consensus is always secured. When the logical interdependencies have a cascade structure, and by considering topics at the top of a cascade structure to be axiom(s) that an individual's belief system is built upon, we establish that discussion of the axiomatic topics will lead to a consensus. In contrast, we discover that persistent disagreement can arise in the topics at the bottom of the cascade when certain types of heterogeneity exist in the logic matrices. A preliminary work [22] considers the special case of lower triangular logic matrices, but we go well beyond that in this paper by considering general logic matrix structures and providing a comprehensive account of the results.

We discover that if there is a failure to reach a consensus, then it is typically not minor; in general a strong diversity of opinions will eventually emerge. In more detail, a network is said to exhibit weak diversity [23] if opinions eventually converge into clusters where there is no difference between opinions in the same cluster (consensus is the special case of one single cluster). Strong diversity occurs when the opinions converge to a configuration of persistent disagreement, with a diverse range of values (there may be clusters of opinions with similar, but not equal, values within a cluster). Weak diversity is a common outcome in the HegselmannKrause model, with the network becoming disconnected into subgroups associated with the clusters. In strongly connected networks, weak diversity also emerges in the Altafini model (specifically polarisation of two opinion clusters) when the network is "structurally balanced". However, sign reversal of some selected edges may destroy the structural balance of the network, causing the opinions to converge to a consensus at an opinion value of zero, indicating that the polarisation phenomenon is not robust to changes in the network structure.

There has been a growing interest to study models which are able to capture the more realistic outcome of strong diversity in networks which remain connected [23], [24]. The DeGroot model shows that social influence in a connected network acts to bring opinions closer together until a consensus is achieved, meaning some other socio-psychological process must be at work to generate strong diversity. The FriedkinJohnsen model attributes strong diversity to an individual's stubborn attachment to his/her initial opinion [13]. In contrast, [25] considers a model where an individual's susceptibility to interpersonal influence is dependent on the individual's current opinion; strong diversity is verified as a special case. The papers [23], [24] consider two features that might give rise to strong diversity, the first being "social distancing", and the second being an individual's "desire to be unique". Experimental studies are inconclusive with regards to the existence of ubiquitous and persistent antagonistic interpersonal interactions (there might be limited occurrences in the network over short time spans) [18], while it is unlikely that an individual has the same level of stubborn attachment to his or her initial opinion value for months or years.

In contrast to these works, we identify for the first time in the literature that strong diversity can arise because of the differences in individuals' belief systems; heterogeneity among belief systems and a cascade logic structure are necessary conditions for strong diversity. In the model, each individual is concurrently undergoing two driver processes; individuallevel belief system dynamics to secure logical consistency of opinions across a set of topics, and interpersonal influence to reach a consensus. Our findings explain that when the two drivers do not interfere with each other, a consensus is reached, whereas conflict between the two drivers leads to persistent disagreement even though all individuals are trying to reach a consensus. This gives a new and illuminating perspective as to why strong diversity can last for extended periods of time in connected networks.

The rest of the paper is structured as follows. In Section II, we provide notations, an introduction to graph theory and the opinion dynamics model. At the same time, a formal problem statement is given. The main results are presented in Section III, with simulations and discussions given in Section IV, and conclusions in Section V.

\section{BACKGROUND AND Formal Problem StATEMENT}

We first introduce some mathematical notations used in the paper. The $(i, j)^{t h}$ entry of a matrix $\boldsymbol{M}$ is denoted $m_{i j}$. A matrix $\boldsymbol{A}$ is said to be nonnegative (respectively positive) if all $a_{i j}$ are nonnegative (respectively positive). We denote $\boldsymbol{A}$ as being nonnegative and positive by $\boldsymbol{A} \geq 0$ and $\boldsymbol{A}>0$, respectively. A matrix $\boldsymbol{A} \geq 0$ is said to be row-stochastic (respectively, row-substochastic) if there holds $\sum_{j=1}^{n} a_{i j}=$ $1, \forall i$ (respectively, if there holds $\sum_{j=1}^{n} a_{i j} \leq 1, \forall i$ and $\exists k$ : $\sum_{j=1}^{n} a_{k j}<1$ ). Let $\mathbf{1}_{n}$ and $\mathbf{0}_{n}$ denote, respectively, the $n \times 1$ column vectors of all ones and all zeros. The $n \times n$ identity matrix is given by $\boldsymbol{I}_{n}$. Two matrices $\boldsymbol{A}$ and $\boldsymbol{B}$ of the same dimension are said to be of the same type, denoted by $\boldsymbol{A} \sim \boldsymbol{B}$, if and only if $a_{i j} \neq 0 \Leftrightarrow b_{i j} \neq 0$. The Kronecker product is denoted by $\otimes$.

\section{A. Graph Theory}

The interaction between $n$ individuals in a social network, and the logical interdependence between topics, can be separately modelled using weighted directed graphs. To that end, we introduce some notation and concepts for graphs. A directed graph $\mathcal{G}[\boldsymbol{A}]=(\mathcal{V}, \mathcal{E}, \boldsymbol{A})$ is a triple where node $v_{i}$ is in the finite, nonempty set of nodes $\mathcal{V}=\left\{v_{1}, \ldots, v_{n}\right\}$. The set of ordered edges is $\mathcal{E} \subseteq \mathcal{V} \times \mathcal{V}$. We denote an ordered edge as $e_{i j}=\left(v_{i}, v_{j}\right) \in \mathcal{E}$, and because the graph is directed, 
in general the existence of $e_{i j}$ does not imply existence of $e_{j i}$. An edge $e_{i j}$ is said to be outgoing with respect to $v_{i}$ and incoming with respect to $v_{j}$. Self-loops are allowed, i.e. $e_{i i}$ may be in $\mathcal{E}$. The matrix $\boldsymbol{A} \in \mathbb{R}^{n \times n}$ associated with $\mathcal{G}[\boldsymbol{A}]$ captures the edge weights. More specifically, $a_{i j} \neq 0$ if and only if $e_{j i} \in \mathcal{E}$. If $\boldsymbol{A}$ is nonnegative, then all edges $e_{i j}$ have positive weights, while a generic $\boldsymbol{A}$ may be associated with a signed graph $\mathcal{G}[\boldsymbol{A}]$, having signed edge weights.

A directed path is a sequence of edges of the form $\left(v_{p_{1}}, v_{p_{2}}\right),\left(v_{p_{2}}, v_{p_{3}}\right), \ldots$ where $v_{p_{i}} \in \mathcal{V}$ are unique, and $e_{p_{i} p_{i+1}} \in \mathcal{E}$. Node $i$ is reachable from node $j$ if there exists a directed path from $v_{j}$ to $v_{i}$. A graph is said to be strongly connected if every node is reachable from every other node. A square matrix $\boldsymbol{A}$ is irreducible if and only if the associated graph $\mathcal{G}[\boldsymbol{A}]$ is strongly connected. A directed cycle is a directed path that starts and ends at the same node, and contains no repeated node except the initial (which is also the final) node. The length of a directed cycle is the number of edges in the directed cyclic path. A directed graph is aperiodic if there exists no integer $k>1$ that divides the length of every directed cycle of the graph [26], and any graph with a self-loop is aperiodic.

A signed graph $\mathcal{G}$ is said to be structurally balanced (respectively structurally unbalanced) if the nodes $\mathcal{V}=\left\{v_{1}, \ldots v_{n}\right\}$ can be partitioned (respectively cannot be partitioned) into two disjoint sets such that each edge between two nodes in the same set has a positive weight, and each edge between nodes in different sets has a negative weight [27].

\section{B. The Multi-Dimensional DeGroot Model}

In this paper, we investigate a recently proposed multidimensional extension to the DeGroot and Friedkin-Johnsen models [18], [19], which considers the simultaneous discussion of logically interdependent topics.

Formally, consider a population of $n$ individuals discussing simultaneously their opinions on $m$ topics, with individual and topic index set $\mathcal{I}=\{1, \ldots, n\}$ and $\mathcal{J}=\{1, \ldots, m\}$, respectively. Individual $i$ 's opinions on the $m$ topics at time $t=0,1, \ldots$, are denoted by $\boldsymbol{x}_{i}(t)=\left[x_{i}^{1}(t), \ldots, x_{i}^{m}(t)\right]^{\top} \in$ $\mathbb{R}^{m}$. In this paper, we adopt a standard definition of an opinion [19]. In particular, $x_{i}^{p}(t) \in[-1,1]$ is individual $i$ 's attitude towards topic $p$, which takes the form of a statement, with $x_{i}^{p}>0$ representing $i$ 's support for statement $p, x_{i}^{p}<0$ representing rejection of statement $p$, and $x_{i}^{p}=0$ representing a neutral stance. The magnitude of $x_{i}^{p}$ denotes the strength of conviction, with $\left|x_{i}^{p}\right|=1$ being maximal support/rejection. Mild assumptions are placed on the network and individual parameters in the sequel to ensure that $x_{i}^{p}(t) \in[-1,1]$ for all $t \geq 0$, and thus the opinion values are always well defined.

The multi-dimensional DeGroot model posits that

$$
\boldsymbol{x}_{i}(t+1)=\sum_{j=1}^{n} w_{i j} \boldsymbol{C}_{i} \boldsymbol{x}_{j}(t),
$$

where the nonnegative scalar $w_{i j}$ represents the influence weight individual $i$ accords to the vector of opinions of individual $j$. Thus, the influence matrix $\boldsymbol{W} \in \mathbb{R}^{n \times n}$, with $(i, j)^{t h}$ entry $w_{i j}$, can be used to define the graph $\mathcal{G}[\boldsymbol{W}]$ that describes the interpersonal influences of the $n$ individuals. We assume that $w_{i i}>0$ and $\sum_{j=1}^{n} w_{i j}=1$ for all $i \in \mathcal{I}$, which implies that $\boldsymbol{W}$ is row-stochastic. In some cases, information between individuals may not be bidirectional; individual $i$ knows the opinions of individual $j$ but not vice versa (e.g. on Twitter, $i$ may follow $j$ but $j$ may not follow $i$ ). Even in the bidirectional case, the weight assigned by $i$ to $j$ 's opinions may not be equal to the weight $j$ assigns to $i$ 's opinions. Thus, it is not necessarily true that $w_{i j}=w_{j i}$, meaning $\boldsymbol{W}$ is not necessarily symmetric and so $\mathcal{G}[\boldsymbol{W}]$ might be directed.

The matrix $\boldsymbol{C}_{i} \in \mathbb{R}^{m \times m}$, with $(p, q)^{t h}$ entry $c_{p q, i}$, is termed the logic matrix. In [18], [19], the authors elucidate that $\boldsymbol{C}_{i}$ represents the logical interdependence between the $m$ topics as seen by individual $i$. We note that in this paper, the $C_{i}$ are assumed to be heterogeneous (i.e. $\exists i, j: \boldsymbol{C}_{i} \neq \boldsymbol{C}_{j}$ ). Indeed, a critical aspect of this paper is to study how the structure of the $C_{i}$ s, especially heterogeneity, can determine whether certain topics have opinions that reach a consensus or a persistent disagreement.

We now illustrate with a simple example how $\boldsymbol{C}_{i}$ is used by individual $i$ to obtain a set of opinions consistent with any logical interdependencies between each topic, and in doing so, motivate that certain constraints must be imposed on $\boldsymbol{C}_{i}$ due to the problem context (these constraints are implicitly imposed in [18], [19]). As will be evident from the following example, logical relationships between two topics $p$ and $q$ are not necessarily symmetric, so it is not necessarily true that $c_{p q, i}=c_{q p, i}$. In fact, it is also possible that $c_{p q, i}=0$ while $c_{q p, i} \neq 0$. Thus, $\boldsymbol{C}$ is not necessarily symmetric and so $\mathcal{G}\left[\boldsymbol{C}_{i}\right]$ might be directed.

Suppose that there are two topics. Topic 1: The exploration of Space is important to mankind's future. Topic 2: The exploration of Space should be privatised. Using Topic 1 as an example, and according to the definition of an opinion given above Eq. (1), $x_{i}^{1}=1$ represents individual $i$ 's maximal support of the importance of Space exploration, while $x_{i}^{1}=-1$ represents maximal rejection that Space exploration is important. Now, suppose that individual $i$ has $\boldsymbol{x}_{i}(0)=[1,-0.2]^{\top}$, i.e. individual $i$ initially believes with maximal conviction that Space exploration is important and initially believes with some (but not absolute) conviction that Space exploration should not be privatised ${ }^{1}$. Let

$$
\boldsymbol{C}_{i}=\left[\begin{array}{cc}
1 & 0 \\
0.5 & 0.5
\end{array}\right]
$$

This tells us that individual $i$ 's opinion on the importance of Space exploration is unaffected by his or her own opinion on whether Space exploration should be privatised. On the other hand, individual $i$ 's opinion on Topic 2 depends positively on his or her own opinion on Topic 1 , perhaps because individual $i$ believes privatised companies are more effective at making breakthrough progress. In the absence of opinions from other individuals, individual $i$ 's opinions evolves as

$$
\boldsymbol{x}_{i}(t+1)=\boldsymbol{C}_{i} \boldsymbol{x}_{i}(t)
$$

\footnotetext{
${ }^{1}$ Note that we do not require $\boldsymbol{C}_{i}$ to be row-stochastic and nonnegative, though the $\boldsymbol{C}_{i}$ of this example is.
} 
which yields $\lim _{t \rightarrow \infty} \boldsymbol{x}_{i}(t)=[1,1]^{\top}$, i.e. individual $i$ eventually believes that Space exploration should be privatised. Thus, $\boldsymbol{x}_{i}(t)$ moves from $\boldsymbol{x}_{i}(0)=[1,-0.2]^{\top}$, where individual $i$ 's opinions are inconsistent with the logical interdependence as captured by $\boldsymbol{C}_{i}$, to the final state $\boldsymbol{x}_{i}(\infty)=[1,1]^{\top}$, which is consistent with the logical interdependence. Eq. (3), with opinion vector $\boldsymbol{x}_{i}(t)$ and the logical interdependencies captured by $\boldsymbol{C}_{i}$, models individual $i$ 's belief system. (We explained qualitatively what a belief system was in the Introduction, and have now given the mathematical formulation.)

In general, one might expect, as do we in this paper, that an individual's belief system without interpersonal influence from neighbours will eventually become consistent. For a topic $p$ which is independent of all other topics, one also expects that $x_{i}^{p}(t+1)=x_{i}^{p}(t)$ for all $t$. To ensure the belief system is eventually consistent, we impose the following assumption.

Assumption 1. For all $i \in \mathcal{I}$, the matrix $\boldsymbol{C}_{i}$ with $(p, q)^{t h}$ entry $c_{p q, i}$, is such that each eigenvalue of $C_{i}$ is either 1 or has modulus less than 1. If an eigenvalue of $\boldsymbol{C}_{i}$ is 1 , then it is semi-simple ${ }^{2}$. For all $i \in \mathcal{I}$ and $p \in \mathcal{J}$, there holds $\sum_{q=1}^{m}\left|c_{p q, i}\right|=1$, and the diagonal entries satisfy $c_{p p, i}>0$.

The assumptions on the eigenvalues of $\boldsymbol{C}_{i}$ are necessary and sufficient [26, Lemma 1.7] for Eq. (3) to converge to a limit, i.e. for individual $i$ 's belief system to eventually become consistent. The other assumptions lead to desirable properties for the system Eq. (1). Specifically, the reasonable assumption that $c_{p p, i}>0$ means topic $p$ is positively correlated with itself. The constraint $\sum_{q=1}^{m}\left|c_{p q, i}\right|=1$ for all $i \in \mathcal{I}$ and $p \in \mathcal{J}$ ensures that if $x_{i}^{p}(0) \in[-1,1]$ for all $i \in \mathcal{I}$ and $p \in \mathcal{J}$, then it is guaranteed that $x_{i}^{p}(t) \in[-1,1]$ for all $t \geq 0$ (this is proved in [18]). From this constraint, we also observe that if topic $p$ is independent of all other topics, i.e. $c_{p q, i}=0$ for all $q \neq p$, then $c_{p p, i}=1$. The well-studied special case where topics are totally independent is $\boldsymbol{C}_{i}=\boldsymbol{I}_{m}$. We are now in a position to formally define this paper's objective.

\section{Objective Statement}

This paper is focused on establishing the effects of the set of logic matrices $\boldsymbol{C}_{i}, i \in \mathcal{I}$ on the evolution of opinions, and in particular the limiting opinion configuration. First, we record two assumptions on the logic matrix and the network topology, which will hold throughout this paper, and then explain the motivation for these assumptions.

\section{Assumption 2. For every $i, j \in \mathcal{I}$, there holds $\boldsymbol{C}_{i} \sim \boldsymbol{C}_{j}$.}

Assumption 3. The influence network $\mathcal{G}[\boldsymbol{W}]$ is strongly connected, $\boldsymbol{W}$ is row-stochastic, and $w_{i i}>0, \forall i \in \mathcal{I}$.

Assumption 2 implies that, for every $i, j \in \mathcal{I}$, the graphs $\mathcal{G}\left[\boldsymbol{C}_{i}\right]$ and $\mathcal{G}\left[\boldsymbol{C}_{j}\right]$ have the same structure (but possibly with different edge weights, including weights of opposing signs). This means that all individuals have the same view on which topics have dependent relationships with which other topics, but the assigned weights $c_{i j}$ (and signs) may be different. This

\footnotetext{
${ }^{2} \mathrm{By}$ semi-simple, we mean that the geometric and algebraic multiplicities are the same. Equivalently, all Jordan blocks of the eigenvalue 1 are 1 by 1 .
}

assumption ensures that the scope of this paper is reasonable, because if $\boldsymbol{C}_{i} \sim \boldsymbol{C}_{j}$ does not hold, the problem complexity explodes due to the large number of different scenarios one needs to analyse.

Assumption 3 is not necessary to establish convergence of Eq. (1). Rather, we deliberately impose Assumption 3 in order to draw an important contrast with existing work. This is because if $\mathcal{G}[\boldsymbol{W}]$ is strongly connected and $\boldsymbol{C}_{i}=\boldsymbol{C}_{j}=$ $C, \forall i, j \in \mathcal{I}$ (homogeneous), the opinions in each topic will reach a consensus. By establishing that disagreement can arise under Assumption 3 (as we will show in Section III), we directly prove that disagreement is due to heterogeneity in $C_{i}$, i.e. heterogeneity in individuals' belief systems. We provide further details and discussions of this particular aspect in Section IV-C.

Objective 1. Let a set of logic matrices $\boldsymbol{C}_{i}, i \in \mathcal{I}$ and an influence network $\mathcal{G}[\boldsymbol{W}]$ be given, satisfying Assumptions 1, 2 and 3. Suppose that each individual $i$ 's opinion vector $\boldsymbol{x}_{i}(t) \in$ $[-1,1]^{m}$ evolves according to Eq. (1). Then, for each $k \in \mathcal{J}$ and generic initial conditions $\boldsymbol{x}(0) \in[-1,1]^{n m}$, this paper will investigate a method to systematically determine when there exists, and when there does not exist, an $\alpha_{k} \in[-1,1]$ such that

$$
\lim _{t \rightarrow \infty} x_{i}^{k}(t)=\alpha_{k}, \forall i \in \mathcal{I} .
$$

We will show with the main theoretical results in Section III that $\boldsymbol{C}_{i}$ of a certain structure always guarantees consensus (i.e. Eq. (4) is satisfied), and conversely, that $\boldsymbol{C}_{i}$ of a certain other structure will lead to disagreement in certain identifiable topics (i.e. Eq. (4) is not satisfied). This will help achieve the above objective. Then, in Section IV we will compare our findings with the existing results to illustrate the unique phenomena that can arise when introducing heterogeneity into the $\boldsymbol{C}_{i}$ matrix, and the novel explanation of disagreement we obtain.

To conclude this subsection, we now provide the definition of "competing logical interdependencies" which will be important in some scenarios for characterising the final opinions.

Definition 1 (Competing Logical Interdependence). An influence network is said to contain individuals with competing logical interdependencies on topic $p \in \mathcal{J}$ if there exist individuals $i, j$ such that for some $q \in \mathcal{J} \backslash\{p\}, \boldsymbol{C}_{i}$ and $\boldsymbol{C}_{j}$ have nonzero entries $c_{p q, i}$ and $c_{p q, j}$ that are of opposite signs.

In other words, individuals with competing logical interdependencies are those who, when having the same opinion on topic $q$, move in opposite directions on the opinion spectrum for topic $p$. Using the example in Section II-B, one might have an individual $j$ with

$$
\boldsymbol{C}_{j}=\left[\begin{array}{cc}
1 & 0 \\
-0.5 & 0.5
\end{array}\right] .
$$

because $j$ considers that private companies are profit-driven, and therefore cannot be ethically trusted with the exploration of Space. Then, from Eq. (3), one has that $\boldsymbol{x}_{j}(\infty)=[1,-1]^{\top}$, i.e. individual $j$ eventually firmly believes Space exploration should not be privatised. In particular, $x_{j}^{1}(\infty)=-x_{j}^{2}(\infty)$.

In light of Assumption 2, if two individuals have competing interdependencies on topic $p$, then for every individual $i \in \mathcal{I}$, 
there is necessarily some individual $k \in \mathcal{I} \backslash\{i\}$ with whom individual $i$ has competing logical interdependence on topic $p$ : the nonzero entries $c_{p q, i}$ and $c_{p q, k}$ are of opposite signs for some $q \in \mathcal{J}$.

Remark 1. Recall that $\boldsymbol{C}_{i}$ is individual i's set of constraints/functional dependencies between topics in i's belief system. Thus, heterogeneity of $\boldsymbol{C}_{i}$ may arise for many different reasons, such as education, background, or expertise in the topic. For example, if the set of topics is related to sports, a professional athlete may have very different weights (including the signs) in $\boldsymbol{C}_{i}$ compared to someone that does not pursue an active lifestyle. Interestingly, [28] showed that when presented with the same published statement on an issue, different people could take opposite positions on the issue.

\section{MAIN RESUlts}

The main results are presented in two parts. First, we establish a general convergence result for the networked system. Then, we analyse the limiting opinion distribution and the role of the set of logic matrices in determining whether opinions for a given topic will reach consensus or fail to do so. In order to focus on the theoretical results and interpretations as social phenomena, all proofs are presented in the Appendix.

\section{A. Convergence}

Denoting the vector of opinions for the entire influence network as $\boldsymbol{x}=\left[\boldsymbol{x}_{1}(t)^{\top}, \ldots, \boldsymbol{x}_{n}(t)^{\top}\right]^{\top} \in \mathbb{R}^{n m}$, the network dynamics of Eq. (1) are given by

$$
\boldsymbol{x}(t+1)=\left[\begin{array}{ccc}
w_{11} \boldsymbol{C}_{1} & \cdots & w_{1 n} \boldsymbol{C}_{1} \\
\vdots & \ddots & \vdots \\
w_{n 1} \boldsymbol{C}_{n} & \cdots & w_{n n} \boldsymbol{C}_{n}
\end{array}\right] \boldsymbol{x}(t),
$$

and we define the system matrix above as $\boldsymbol{B} \in \mathbb{R}^{n m \times n m}$. To begin, we rewrite the network dynamics Eq. (6) into a different form to aid analysis by introducing a reordering.

For all $k \in \mathcal{J}$, define $\boldsymbol{y}_{k}(t)=\left[y_{k}^{1}(t), \ldots y_{k}^{n}(t)\right]^{\top}=$ $\left[x_{1}^{k}(t), \ldots, x_{n}^{k}(t)\right]^{\top}$. In other words, for given $i \in \mathcal{I}$ and $k \in$ $\mathcal{J}, y_{k}^{i}$ represents individual $i$ 's opinion on topic $k$. The reader is also referred to Fig. 1 for an example of the reordering. Then, $\boldsymbol{y}_{k}(t) \in \mathbb{R}^{n}$ is the vector of all $n$ individuals' opinions on the $k^{t h}$ topic at time $t$, and we say that the opinions on topic $k$ are at a consensus if $\boldsymbol{y}_{k}=\alpha \mathbf{1}_{n}$ for some scalar $\alpha$. For all $k, j \in \mathcal{J}$, we define $\boldsymbol{\Gamma}_{k j}=\operatorname{diag}\left(c_{k j, 1}, \ldots, c_{k j, n}\right) \in \mathbb{R}^{n \times n}$ as the diagonal matrix with the $i^{t h}$ diagonal element being $c_{k j, i}$, the $(k, j)^{t h}$ entry of $\boldsymbol{C}_{i}$. One obtains that

$$
\boldsymbol{y}_{k}(t+1)=\sum_{j=1}^{m} \boldsymbol{\Gamma}_{k j} \boldsymbol{W} \boldsymbol{y}_{j}(t)
$$

Defining $\boldsymbol{y}(t)=\left[\boldsymbol{y}_{1}(t)^{\top}, \ldots, \boldsymbol{y}_{m}(t)^{\top}\right]^{\top} \in \mathbb{R}^{n m}$, we further obtain

$$
\boldsymbol{y}(t+1)=\left[\begin{array}{ccc}
\boldsymbol{\Gamma}_{11} \boldsymbol{W} & \cdots & \boldsymbol{\Gamma}_{1 m} \boldsymbol{W} \\
\vdots & \ddots & \vdots \\
\boldsymbol{\Gamma}_{m 1} \boldsymbol{W} & \cdots & \boldsymbol{\Gamma}_{m m} \boldsymbol{W}
\end{array}\right] \boldsymbol{y}(t)
$$

We denote the matrix in Eq. (8) as $\boldsymbol{A} \in \mathbb{R}^{n m \times n m}$, with block matrix elements $\boldsymbol{A}_{p q}=\boldsymbol{\Gamma}_{p q} \boldsymbol{W} \in \mathbb{R}^{n \times n}$. We now show how
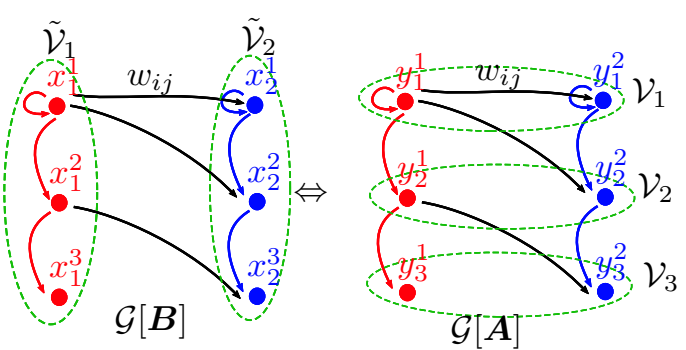

Figure 1. An illustrative network with 2 individuals discussing 3 topics, with only selected edges drawn for clarity. Each node represents the opinion of an individual for a topic, with red and blue nodes associated with individuals 1 and 2, respectively. The black edges represent interpersonal influence via the weight $w_{i j}$, while the coloured edges represent logical interdependencies between topics. In $\mathcal{G}[\boldsymbol{B}]$, nodes are grouped and ordered by individual in node subset $\tilde{\mathcal{V}}_{q}$ (as illustrated by the dotted green ellipse groupings) leading to Eq. (6). In $\mathcal{G}[\boldsymbol{A}]$, the nodes are grouped and ordered by topic in node subset $\mathcal{V}_{p}$ (as illustrated by the dotted green ellipses) leading to Eq. (8).

the system Eq. (8) can be considered as a consensus process on a multiplex (or multi-layered) signed graph.

Consider the matrix $\boldsymbol{A}$ in Eq. (8), with the associated graph $\mathcal{G}[\boldsymbol{A}]$, and the matrix $\boldsymbol{B}$ in Eq. (6), with associated graph $\mathcal{G}[\boldsymbol{B}]$. Clearly, the two graphs are the same up to a reordering of the nodes. In $\mathcal{G}[\boldsymbol{A}]$, with node set $\mathcal{V}[\boldsymbol{A}]=\left\{v_{1}, \ldots, v_{n m}\right\}$, one can consider the node subset $\mathcal{V}_{p}=\left\{v_{(p-1) n+1}, \ldots, v_{p n}\right\}$, $p \in \mathcal{J}$ as a layer of the multi-layer graph $\mathcal{G}[\boldsymbol{A}]$ with vertices associated with the opinions of individuals $1, \ldots, n$ on topic $p$. In $\mathcal{G}[\boldsymbol{B}]$, with node set $\mathcal{V}[\boldsymbol{B}]=\left\{v_{1}, \ldots, v_{n m}\right\}$, one can consider the node subset $\tilde{\mathcal{V}}_{q}=\left\{v_{(q-1) m+1}, \ldots, v_{q m}\right\}, q \in \mathcal{I}$ as a layer of a multi-layer graph with vertices associated with the opinions of individual $q$ on topics $1, \ldots, m$. This is illustrated in Fig. 1, where each layer is identified by a dotted green ellipse border. A key motivation to study $\mathcal{G}[\boldsymbol{A}]$ and the dynamical system Eq. (8) is that all the block diagonal entries $\boldsymbol{A}_{i i}$ of $\boldsymbol{A}$ are nonnegative and irreducible because Assumption 1 indicates that $\boldsymbol{\Gamma}_{p p}$ is a positive diagonal matrix. This means that the edges between nodes in the subset $\mathcal{V}_{p}=\left\{v_{(p-1) n+1}, \ldots, v_{p n}\right\}, p \in \mathcal{J}$ have positive weights, a property which greatly aids in the checking of the structural balance or unbalance of $\mathcal{G}[\boldsymbol{A}]$ given $\mathcal{G}[\boldsymbol{W}]$ and $\boldsymbol{C}_{i}, \forall i \in \mathcal{I}$.

Verify from the row-stochastic property of $\boldsymbol{W}$ and the rowsum property of $\boldsymbol{C}_{i}$ in Assumption 1 that the entries of $\boldsymbol{A}$ satisfy $\sum_{q=1}^{n m}\left|a_{p q}\right|=1$ for all $p=1, \ldots, n m$. One can conclude that Eq. (8) has the same dynamics as the discretetime Altafini model (see e.g. [9], [10]).

Remark 2. Although Eq. (8) has the same dynamics as the discrete-time Altafini model, a number of important differences exist. First, the context of negative edge weights is entirely different: in the Altafini model, $w_{i j}<0$ implies individual $i$ mistrusts individual $j$ [9]. In contrast, Eq. (8) assumes nonnegative influence $w_{i j} \geq 0$, and the negative edge weights arise from negative logical interdependencies in $\boldsymbol{C}_{i}$. Moreover the network structure of $\mathcal{G}[\boldsymbol{A}]$ is affected by both the influence network $\mathcal{G}[\boldsymbol{W}]$ and the logic matrix graphs $\mathcal{G}\left[\boldsymbol{C}_{i}\right]$.

The main convergence result is given as follows.

Theorem 1. Suppose that for a population of $n$ individuals, the vector of the $n$ individuals' opinions $\boldsymbol{y}(t)$ evolves 
according to Eq. (8), with interpersonal influences captured by $\mathcal{G}[\boldsymbol{W}]$. Suppose further that Assumptions 1, 2, and 3 hold. Then, for any initial condition $\boldsymbol{y}(0) \in[-1,1]^{n m}$, there exists some $\boldsymbol{y}^{*} \in[-1,1]^{n m}$ such that there holds $\lim _{t \rightarrow \infty} \boldsymbol{y}(t)=\boldsymbol{y}^{*}$ exponentially fast.

We remark that for arbitrary initial conditions $\boldsymbol{y}(0) \in \mathbb{R}^{n m}$ one can still prove that $\lim _{t \rightarrow \infty} \boldsymbol{y}(t)=\boldsymbol{y}^{*}$ for some $\boldsymbol{y}^{*}$. However, $\boldsymbol{y}^{*} \in[-1,1]^{n m}$ is no longer guaranteed, and thus to keep consistent with the rest of the paper, the statement in Theorem 1 assumes $\boldsymbol{y}(0) \in[-1,1]^{n m}$. Having established that the opinion dynamical system always converges, we turn to addressing Objective 1 by studying the influence of $\boldsymbol{C}_{i}$ in determining the limiting opinion vector $\boldsymbol{y}^{*}$.

\section{B. Consensus and Disagreement of Each Topic}

We now explain how to use the logic matrices $\boldsymbol{C}_{i}$ to systematically determine whether opinions on a given topic $p \in \mathcal{J}$ will reach a consensus or not. In Section IV, we present simulations and discussions to illustrate how to use our results, and to highlight interesting social interpretations of the theoretical results.

Consider the graph $\mathcal{G}\left[\boldsymbol{C}_{i}\right]$ associated with $\boldsymbol{C}_{i}$ for some $i \in \mathcal{I}$, which is a signed graph if there are negative offdiagonal entries in $\boldsymbol{C}_{i}$. Under Assumption 2, irreducibility of one $\boldsymbol{C}_{i}$ implies the same for all, and in the absence of competing logical interdependencies, the structural balance or unbalance of one $\mathcal{G}\left[\boldsymbol{C}_{i}\right]$ implies the same for all. Irreducible logic matrices correspond to strongly connected $\mathcal{G}\left[\boldsymbol{C}_{i}\right]$, meaning all topics are directly or indirectly dependent on all other topics. We now present a theorem establishing that if $\boldsymbol{C}_{i}$ for all $i \in \mathcal{I}$ are irreducible, then all topics will reach a consensus (although the consensus value for two different topics $p$ and $q$ may be different).

Theorem 2. Let the hypotheses in Theorem 1 hold. Suppose that $\boldsymbol{y}(0) \in[-1,1]^{n m}$ and Assumptions 1, 2, and 3 hold. Suppose further that $\boldsymbol{C}_{i}, \forall i \in \mathcal{I}$ are irreducible. Then, for all $k \in \mathcal{J}, \lim _{t \rightarrow \infty} \boldsymbol{y}_{k}(t)=\alpha_{k} \mathbf{1}_{n}$ exponentially fast, for some $\alpha_{k} \in[-1,1]$. Moreover,

1) If there are no competing logical interdependencies, as given in Definition 1 , and $\mathcal{G}\left[\boldsymbol{C}_{i}\right], \forall i \in \mathcal{I}$ are structurally balanced, then for almost all initial conditions, $\left|\alpha_{p}\right|=$ $\left|\alpha_{q}\right| \neq 0, \forall p, q \in \mathcal{J}$.

2) If either (i) $\mathcal{G}\left[\boldsymbol{C}_{i}\right], \forall i \in \mathcal{I}$ are structurally unbalanced, or (ii) there are competing logical interdependencies, then $\alpha_{k}=0, \forall k \in \mathcal{J}$.

Further to the conclusions of Theorem 2, one can obtain the following result for the case where consensus to a nonzero opinion value is achieved.

Corollary 1. Let the hypotheses in Theorem 2 hold. Suppose that there are no competing logical interdependencies, and $\mathcal{G}\left[\boldsymbol{C}_{i}\right], \forall i \in \mathcal{I}$ are structurally balanced. For $\mathcal{G}\left[\boldsymbol{C}_{i}\right]$ with node set $\mathcal{V}=\left\{v_{1}, \ldots, v_{m}\right\}$, define two disjoint subsets of nodes $\mathcal{V}\left[\boldsymbol{C}_{i}\right]^{+}$and $\mathcal{V}\left[\boldsymbol{C}_{i}\right]^{-}$so that each edge between two nodes in $\mathcal{V}\left[\boldsymbol{C}_{i}\right]^{+}$or two nodes in $\mathcal{V}\left[\boldsymbol{C}_{i}\right]^{-}$has a positive weight, and each edge between two nodes in $\mathcal{V}\left[\boldsymbol{C}_{i}\right]^{+}$and $\mathcal{V}\left[\boldsymbol{C}_{i}\right]^{-}$has a negative weight. Then, for any $p, q \in \mathcal{J}$, there holds

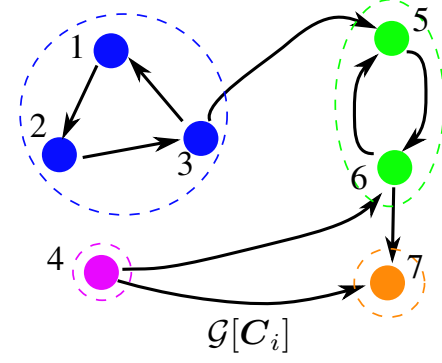

Figure 2. An illustrative example of $\mathcal{G}\left[\boldsymbol{C}_{i}\right]$, with each node representing a topic, and edges representing logical interdependencies between topics (selfloops are hidden for clarity). One can divide the nodes into strongly connected components (each dotted coloured circle denotes a strongly connected component). The results of this paper allow one to progressively analyse each component to establish which topics will have opinions reaching a consensus and which topics will have opinions reaching a persistent disagreement.

$$
\begin{aligned}
& \alpha_{p}=\alpha_{q} \text { if } v_{q}, v_{p} \in \mathcal{V}\left[\boldsymbol{C}_{i}\right]^{+} \text {or } v_{q}, v_{p} \in \mathcal{V}\left[\boldsymbol{C}_{i}\right]^{-} \text {. } \\
& \text { 2) } \alpha_{p}=-\alpha_{q} \text { if } v_{q} \in \mathcal{V}\left[\boldsymbol{C}_{i}\right]^{+} \text {and } v_{p} \in \mathcal{V}\left[\boldsymbol{C}_{i}\right]^{-} .
\end{aligned}
$$

Consider now the more general case where $C_{i} \forall i \in \mathcal{I}$ is reducible, i.e. $\mathcal{G}\left[\boldsymbol{C}_{i}\right]$ is no longer strongly connected. From a graphical perspective, the graph $\mathcal{G}\left[\boldsymbol{C}_{i}\right]$ can be divided into strongly connected components which are "closed" or "open". (This is related to a concept called the condensation of a graph, see [26]). Formally, we say that a subgraph $\overline{\mathcal{G}}$ is a strongly connected component of $\mathcal{G}$ if $\overline{\mathcal{G}}$ is strongly connected and any other subgraph of $\mathcal{G}$ strictly containing $\overline{\mathcal{G}}$ is not strongly connected. A strongly connected component $\overline{\mathcal{G}}$ of a graph $\mathcal{G}$ is said to be closed if there are no incoming edges to $\overline{\mathcal{G}}$ from a node outside of $\overline{\mathcal{G}}$, and is said to be open otherwise. The smallest strongly connected component is a single node, and it would be closed if there are no incoming edges to it. Figure 2 shows an example of a graph $\mathcal{G}\left[\boldsymbol{C}_{i}\right]$ divided into strongly connected components (identified by the encircling dotted lines), with the blue and purple components being closed, and the green and orange components being open.

From an algebraic perspective. Assumption 2 indicates that there exists a common permutation matrix $\boldsymbol{P}$ such that, for all $i \in \mathcal{I}, \boldsymbol{P}^{T} \boldsymbol{C}_{i} \boldsymbol{P}$ is lower block triangular (equivalent to a reordering of the nodes of $\mathcal{G}\left[\boldsymbol{C}_{i}\right]$ ). Without loss of generality, we therefore assume that the topics $p \in \mathcal{J}$ are ordered such that, for each $i \in \mathcal{I}$,

$$
\boldsymbol{C}_{i}=\left[\begin{array}{cccc}
\boldsymbol{C}_{11, i} & \mathbf{0} & \cdots & \mathbf{0} \\
\boldsymbol{C}_{21, i} & \boldsymbol{C}_{22, i} & \cdots & \mathbf{0} \\
\vdots & \vdots & \ddots & \vdots \\
\boldsymbol{C}_{s 1, i} & \boldsymbol{C}_{s 2, i} & \cdots & \boldsymbol{C}_{s s, i}
\end{array}\right]
$$

where $C_{j j, i} \in \mathbb{R}^{s_{j} \times s_{j}}$ is irreducible for any $j \in \mathcal{S} \triangleq$ $\{1,2, \cdots, s\}$ and $s_{j}$ are positive integers such that $\sum_{j=1}^{s} s_{j}=$ $m$. Each $\mathcal{G}\left[\boldsymbol{C}_{j j, i}\right]$ corresponds to a strongly connected component, and there are $s$ components in total, with the $j^{\text {th }}$ component having $s_{j}$ nodes (topics). Decompose the opinion set $\mathcal{J}$ into $s$ disjoint subsets $\mathcal{J}_{j}$ for $j \in \mathcal{S}$ where

$$
\mathcal{J}_{j} \triangleq\left\{\sum_{i=1}^{j} s_{i-1}+1, \sum_{i=1}^{j} s_{i-1}+2, \ldots, \sum_{i=1}^{j} s_{i-1}+s_{j}\right\},
$$


with $s_{0}=0$. To clarify, we now use the example in Fig. 2 to illustrate the notation in Eq. (9) and Eq. (10). First, $\mathcal{S}$ indexes the strongly connected components of $\mathcal{G}\left[\boldsymbol{C}_{i}\right]$ : in Fig. 2, one has $\mathcal{S}=\{$ blue $\triangleq 1$, purple $\triangleq 2$, green $\triangleq 3$, orange $\triangleq 4\}$. Thus, there are $s=4$ strongly connected components, and the number of nodes in the $j^{\text {th }}$ component is $s_{j}$ : we get $s_{1}=$ $3, s_{2}=1, s_{3}=2, s_{4}=1$. Since $\mathcal{J}_{j}$ indexes the topics in the $j^{\text {th }}$ component, one has $\mathcal{J}_{1}=\{1,2,3\}, \mathcal{J}_{2}=\{4\}, \mathcal{J}_{3}=$ $\{5,6\}, \mathcal{J}_{4}=\{7\}$.

Though reducible $\boldsymbol{C}_{i}$ may seem to be restrictive, they are in fact common given the problem context since they imply a cascade logical interdependence structure among the topics. This may be representative of an individual $i$ who obtains $\boldsymbol{C}_{i}$ by sequentially building upon an axiom or axioms (the first $\boldsymbol{C}_{j j, i}$ block matrices corresponding to closed strongly connected components). The two topics of the Space exploration example given in Eq. (2) constitute one such example of a belief system driven by an axiom (Topic 1).

If the topic set $\mathcal{J}_{j}$ corresponds to a closed strongly connected component of $\mathcal{G}\left[\boldsymbol{C}_{i}\right]$, then clearly in Eq. (9), $\boldsymbol{C}_{p j, i}=\mathbf{0}$ for all $p \neq j$ (e.g. the blue and purple components in Fig. 2). Theorem 2 and Corollary 1 establish that for every $k \in \mathcal{J}_{j}$, there holds $\lim _{t \rightarrow \infty} \boldsymbol{y}_{k}(t)=\alpha_{k} \mathbf{1}_{n}$ exponentially fast, with $\alpha_{k} \in[-1,1]$. That is, all opinions in topic $k \in \mathcal{J}_{j}$ reach a consensus. If, on the other hand, the topic set $\mathcal{J}_{j}$ corresponds to an open strongly connected component of $\mathcal{G}\left[\boldsymbol{C}_{i}\right]$ (green and orange components in Fig. 2), then the results we present below can be employed sequentially in order to establish whether opinions on a given topic have reached a consensus. By "sequentially", we mean that we analyse the topic sets $\mathcal{J}_{j}$ with $j$ in the order $1,2, \ldots, s$. Under Assumption 2, define for each topic $p \in \mathcal{J}$, the set

$$
\hat{\mathcal{J}}_{p} \triangleq\left\{q \in \mathcal{J}: c_{p q, i} \neq 0, q \neq p\right\}
$$

where $c_{p q, i}$ is the $p q^{t h}$ entry of $\boldsymbol{C}_{i}$. In other words, $\hat{\mathcal{J}}_{p}$ identifies all topics $q \in \mathcal{J}$ that topic $p$ is logically dependent upon. Because of Assumption 2, the set $\hat{\mathcal{J}}_{p}$ is the same for all individuals $i \in \mathcal{I}$. For example, in Fig. $2, \hat{J}_{6}=\{4,5\}$ because Topic 6 depends on Topics 4 and 5 .

Considering a $\mathcal{J}_{j}$ corresponding to an open strongly connected component of $\mathcal{G}\left[\boldsymbol{C}_{i}\right]$, one can derive from Eq. (8) and Theorem 1 that for all $p \in \mathcal{J}_{j}$, the final opinions are given by

$$
\lim _{t \rightarrow \infty} \boldsymbol{y}_{p}(t) \triangleq \boldsymbol{y}_{p}^{*}=\left(\boldsymbol{I}_{n}-\boldsymbol{\Gamma}_{p p} \boldsymbol{W}\right)^{-1}\left(\sum_{j \in \hat{\mathcal{J}}_{p}} \boldsymbol{\Gamma}_{p j} \boldsymbol{W} \boldsymbol{y}_{j}^{*}\right)
$$

For further details, including the existence of $\left(\boldsymbol{I}_{n}-\boldsymbol{\Gamma}_{p p} \boldsymbol{W}\right)^{-1}$, see the Appendix. To more precisely characterise $\boldsymbol{y}_{p}^{*}$ in Eq. (12) and to help answer Objective 1, we now present two theorems for necessary and sufficient conditions that ensure every topic in the subset $\mathcal{J}_{j}$ reaches a consensus of opinions. The first theorem considers the case when the subset $\mathcal{J}_{j}$ is a singleton (e.g. $\mathcal{J}_{4}=\{7\}$ in Fig. 2), and the second the case when $\mathcal{J}_{j}$ has at least two elements (e.g. $\mathcal{J}_{3}=\{5,6\}$ in Fig. 2).

Theorem 3. Let the hypotheses in Theorem 1 hold. Assume that $\boldsymbol{y}(0) \in[-1,1]^{n m}$ and $\boldsymbol{C}_{i}, \forall i \in \mathcal{I}$ is decomposed as in Eq. (9). Suppose that $\mathcal{J}_{j}=\{p\}$, as defined in Eq. (10), is a singleton, and let $\hat{\mathcal{J}}_{p}$ as defined in Eq. (11) be nonempty.
Suppose further that all topics $q \in \hat{\mathcal{J}}_{p}$ satisfy $\lim _{t \rightarrow \infty} \boldsymbol{y}_{q}=$ $\alpha_{q} \mathbf{1}_{n}, \alpha_{q} \in[-1,1]$. Then, $\lim _{t \rightarrow \infty} \boldsymbol{y}_{p}(t)=\alpha_{p} \mathbf{1}_{n}$ for some $\alpha_{p} \in[-1,1]$ if and only if $\exists \kappa_{p} \in[-1,1]$ such that

$$
\kappa_{p}\left(\boldsymbol{I}_{n}-\boldsymbol{\Gamma}_{p p}\right)=\sum_{q \in \hat{\mathcal{J}}_{p}} \alpha_{q} \boldsymbol{\Gamma}_{p q}
$$

holds. If such a $\kappa_{p}$ exists, then $\alpha_{p}=\kappa_{p}$.

The key necessary and sufficient condition of Eq. (13) is somewhat complex and nonintuitive. Roughly speaking, Theorem 3 states that if there exists $\kappa_{p} \in[-1,1]$ satisfying Eq. (13) then Topic $p$ will reach a consensus value of $\alpha_{p}=\kappa_{p}$. If such a $\kappa_{p}$ does not exist, then Topic $p$ will not reach a consensus. Since $\boldsymbol{\Gamma}_{p p}$ is a positive diagonal matrix with diagonal entries less than 1, it is obvious that $\boldsymbol{I}_{n}-\boldsymbol{\Gamma}_{p p}$ is invertible, and any $\kappa$ satisfying Eq. (13) must be unique. Example 1 in Section IV-A details separate simulation examples in which $\exists \kappa_{p} \in[-1,1]$, and $\nexists \kappa_{p} \in[-1,1]$, satisfying Eq. (13). The following corollary studies Eq. (13) for some situations which are important or of interest in the social context, with Items 1) and 4) illustrated in Section IV-A, Example 1.

Corollary 2. Adopting the hypotheses in Theorem 3, the following hold:

1) Suppose that $\hat{\mathcal{J}}_{p}=\{q\}$ is a singleton. Then, $\exists \kappa_{p} \in$ $[-1,1]$ satisfying Eq. (13) if and only if there do not exist individuals $i, j \in \mathcal{I}$ with competing logical interdependencies on topic $p$.

2) If $\alpha_{q}=0$ for all $q \in \hat{\mathcal{J}}_{p}$, then $\kappa_{p}=0$ satisfies Eq. (13).

3) Suppose that $\hat{\mathcal{J}}_{p}=\left\{q_{1}, \ldots, q_{r}\right\}, r \geq 2$. If $c_{p q_{k}, i}=$ $c_{p q_{k}, j}=c_{p q_{k}}$ for all $k \in\{1, \ldots, r\}$ and $i, j \in \mathcal{I}$, then there exists a $\kappa_{p} \in[-1,1]$ satisfying Eq. (13).

4) Suppose that $\hat{\mathcal{J}}_{p}=\left\{q_{1}, \ldots, q_{r}\right\}, r \geq 2$. Suppose further that $\left|\alpha_{q_{u}}\right|=\left|\alpha_{q_{v}}\right|$ for all $u, v \in\{1, \ldots, r\}$. Then, there exists a $\kappa_{p} \in[-1,1]$ satisfying Eq. (13) if either (i) the sign of $c_{p q_{k}, i}$ and $\alpha_{q_{k}}$ are equal for all $i \in \mathcal{I}$ and $k \in$ $\{1, \ldots, r\}$ or (ii) the sign of $c_{p q_{k}, i}$ and $\alpha_{q_{k}}$ are opposite for all $i \in \mathcal{I}$ and $k \in\{1, \ldots, r\}$. In the case of $(i)$, $\kappa_{p}=\left|\alpha_{q_{k}}\right|$, and in the case of (ii), $\kappa_{p}=-\left|\alpha_{q_{k}}\right|$.

When $\mathcal{J}_{j}$ is not a singleton, the analysis becomes significantly more involved. To that end, we first introduce some additional notation. Define

$$
\tilde{\mathcal{J}}_{j} \triangleq \cup_{k \in \mathcal{J}_{j}} \hat{\mathcal{J}}_{k} \backslash \mathcal{J}_{j}
$$

as the set of topics not in $\mathcal{J}_{j}$ that the topics in $\mathcal{J}_{j}$ depend upon. For example, in Fig. $2, \mathcal{J}_{3}=\{5,6\}$ (the third strongly connected component consists of Topics 5 and 6), while $\hat{\mathcal{J}}_{5}=$ $\{3,6\}$ (Topic 5 depends on Topics 3 and 6) and $\hat{\mathcal{J}}_{6}=\{4,5\}$ (Topic 6 depends on Topics 4 and 5). Thus, $\tilde{\mathcal{J}}_{3}=\{3,4\}$ because outside of the strongly connected component formed by Topics 5 and 6, Topics 5 and 6 together depend also on Topics 3 and 4 . Note that if $\mathcal{J}_{j}=\{p\}$ is a singleton, we have $\tilde{\mathcal{J}}_{j}=\hat{\mathcal{J}}_{p}$. Perhaps unsurprisingly, Theorem 3 requires that consensus must first occur for topics in $\tilde{\mathcal{J}}_{j}=\hat{\mathcal{J}}_{p}$, on which the topics in $\mathcal{J}_{j}$ depend. The following theorem also has the requirement that consensus occur for all topics in $\tilde{\mathcal{J}}_{j}$.

Theorem 4. Let the hypotheses in Theorem 1 hold. Assume that $\boldsymbol{y}(0) \in[-1,1]^{n m}$, and $\boldsymbol{C}_{i}, \forall i \in \mathcal{I}$ is decomposed as in 
Eq. (9). Suppose that $\mathcal{J}_{j}=\left\{j_{1}, \ldots, j_{z}\right\}$, has $z \geq 2$ elements. Let $\tilde{\mathcal{J}}_{j}$, as defined in Eq. (14), be nonempty and suppose further that all topics $q \in \tilde{\mathcal{J}}_{j}$ satisfy $\boldsymbol{y}_{q}^{*}=\alpha_{q} \mathbf{1}_{n}, \alpha_{q} \in[-1,1]$. Then, $\lim _{t \rightarrow \infty} \boldsymbol{y}_{k}=\alpha_{k} \mathbf{1}_{n}$ for all $k \in \mathcal{J}_{j}$ if and only if there exists a vector $\phi=\left[\phi_{1}, \ldots, \phi_{z}\right]^{\top}$, with $\phi_{k} \in[-1,1] \forall k=$ $1, \ldots, z$, such that

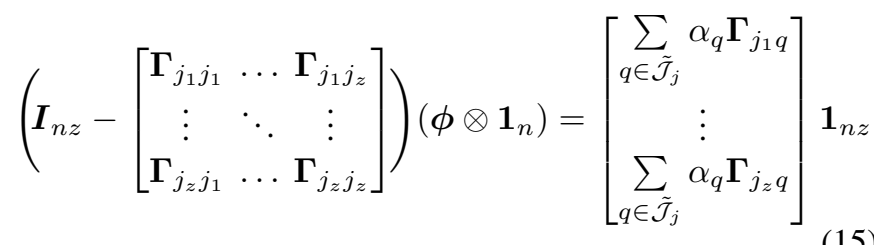

holds. If such a vector $\phi$ exists, then $\alpha_{k}=\phi_{k}$ for all $k \in \mathcal{J}_{j}$.

In Section IV-A, Example 2, we give a working example for how one may obtain $\phi$. Roughly speaking, Theorem 4 states that all topics $j_{1}, \ldots, j_{z}$ of the set $\mathcal{J}_{j}$ will reach a consensus if there exists a $\phi$, with each entry having modulus equal to 1 or less, satisfying Eq. (15). If such a $\phi$ does not exist, then all topics in $\mathcal{J}_{j}$ will fail to reach a consensus. The matrix on the left of Eq. (15) is invertible (see Appendix G), which implies that if $\phi$ exists satisfying Eq. (15), then it is unique. Similar to above, we now present a corollary which gives sufficient conditions for Eq. (15) in two scenarios.

Corollary 3. Adopting the hypotheses in Theorem 4, the following hold:

1) If $\alpha_{q}=0$ for all $q \in \tilde{\mathcal{J}}_{j}$, then $\phi=\mathbf{0}_{z}$ satisfies Eq. (15).

2) If $c_{k p, i}=c_{k p, h}$ for all $k \in \mathcal{J}_{j}, p \in \mathcal{J}$ and $i, h \in \mathcal{I}$, then there exists a $\phi$ satisfying Eq. (15).

For any given set of logic matrices $C_{i}$ and strongly connected social network $\mathcal{G}[\boldsymbol{W}]$, Theorems 2, 3, 4 together establish comprehensive necessary and sufficient conditions enabling us to determine whether a consensus of opinions is reached for every topic $k \in \mathcal{J}$. Since the theorems also detail necessary conditions, we are also able to establish when disagreement arises, because if the opinions for a given topic are not at a consensus, then by definition the opinions are at a disagreement. Objective 1 has been achieved. For the illustrative example in Fig. 2, one would first analyse the blue and purple components using Theorem 2. Then, one would analyse the green component using Theorem 4, and last the orange component using Theorem 3 .

\section{Simulations And Discussions}

In Section IV-A we use several simulation examples to illustrate select key conclusions of Section III, and then provide social interpretations of our results in Section IV-B. Section IV-C provides a comparison and discussion of our findings relative to other existing works in the literature.

\section{A. Simulation Examples}

We now provide several simulations to illustrate some of the results in Section III using a network $\mathcal{G}[\boldsymbol{W}]$ of $n=6$ individuals, with a $\boldsymbol{W}$ which satisfies Assumption 3 (the numerical values of $\boldsymbol{W}$ can be found in the arXiv version this paper [29]). Initial conditions are generated by selecting each $x_{i}^{p}(0)$ from a uniform distribution in $[-1,1]$, and for each simulation example, the same initial conditon vector $\boldsymbol{x}(0)$ is used. This is to isolate and highlight the role of the $\boldsymbol{C}_{i}$ matrix. As will be apparent below, certain entries of $\boldsymbol{C}_{i}$ will be drawn from random uniform distributions, and in all such cases, normalisation of the entries is conducted to ensure the row sum constraint in Assumption 1 holds. The examples will help to explain the two key conditions Eq. (13) and Eq. (15) in Theorems 3 and 4, respectively, while also illustrating that certain qualitative phenomena, viz. reaching consensus or disagreement, do not depend on the precise values of the entries of $\boldsymbol{C}_{i}$.

Example 1: We illustrate Theorem 3 and Eq. (13) with an example of 3 topics, i.e. $\mathcal{J}=\{1,2,3\}$. For all $i=1, \ldots, 6$, the logic matrix is

$$
\boldsymbol{C}_{i}=\left[\begin{array}{ccc}
1 & 0 & 0 \\
-\beta_{i} & 1-\beta_{i} & 0 \\
\delta_{i} & -\eta_{i} & 1-\left(\delta_{i}+\eta_{i}\right)
\end{array}\right]
$$

where $\beta_{i}, \delta_{i}$, and $\eta_{i}$ are drawn from a uniform distribution in the interval $(0,1)$. Then, $\delta_{i}$ and $\eta_{i}$ are appropriately normalised. Notice that there are no competing logical interdependencies associated with the set of $\boldsymbol{C}_{i}$, and according to the notation of Eq. (10), we have $\mathcal{J}_{1}=\{1\}$ (closed), $\mathcal{J}_{2}=\{2\}$ (open), and $\mathcal{J}_{3}=\{3\}$ (open). The temporal evolution of $\boldsymbol{x}(t)$ is given in Fig. 3, with each line denoting an individual's opinion, and different colours denoting different topics.

Consistent with Theorem 2, Topic 1 reaches a consensus, with a specific value in this example of $\alpha_{1}=-0.3484$. Consider now Topic 2, for which Theorem 3 is the relevant result. Here, $p=2$, and $\hat{\mathcal{J}}_{p}=1$ (Topic 2 depends on Topic 1). Because $c_{21, i}$ is negative and $1-c_{22, i}=\left|c_{21, i}\right|$ for all $i$, we have $\boldsymbol{I}_{n}-\boldsymbol{\Gamma}_{22}=-\boldsymbol{\Gamma}_{21}$. Obviously, $\kappa_{2}=0.3484=-\alpha_{1}$ satisfies Eq. (13), and Theorem 3 then establishes that Topic 2 will reach a consensus value of $\alpha_{2}=\kappa_{2}$ (one can also see that Corollary 2 Item 1) applies). One can see this indeed reflected in Fig. 3. Next, we consider Topic 3, with again Theorem 3 being relevant. Now, $p=3$ and $\hat{\mathcal{J}}_{p}=\{1,2\}$ (Topic 3 depends on Topics 1 and 2$)$. Notice that $\operatorname{sign}\left(c_{31, i}\right)=-\operatorname{sign}\left(\alpha_{1}\right)$ and $\operatorname{sign}\left(c_{32, i}\right)=-\operatorname{sign}\left(\alpha_{2}\right)$ for all $i$. Moreover, recall that $1-c_{33, i}=\left|c_{31, i}\right|+\left|c_{32, i}\right|$ for all $i$. One can verify that for $p=3, \kappa_{3}=-0.3484$ satisfies Eq. (13), from which Theorem 3 establishes that Topic 2 will reach a consensus value of $\alpha_{3}=\kappa_{3}$ (see also Corollary 2 Item 4). This can be observed in Fig. 3. It is important to stress that although $\alpha_{1}$ depends on $\boldsymbol{x}(0)$, the $\boldsymbol{C}_{i}$ in Eq. (16) have entries with certain sign patterns which ensure the existence of $\kappa_{2}, \kappa_{3} \in[-1,1]$ satisfying Eq. (13), for all $\alpha_{1} \in[-1,1]$. That is, the consensus of Topics 2 and 3 are robust to initial condition values $\boldsymbol{x}(0)$ and separately to the entries of $\boldsymbol{C}_{i}$ having values randomly drawn from a uniform distribution.

To illustrate the impact of competing logical interdependencies, we make a single and simple change to the simulation setup. Specifically, for $i=1$, we introduce competing logical interdependencies in Topic 2 by changing the $c_{21,1}$ entry of $\boldsymbol{C}_{1}$ from $-\beta_{1}$ to $\beta_{1}$ (from negative to positive weight). The temporal evolution of the opinions is given in Fig. 4. With this adjustment, for Topic 2, there does not exist a $\kappa_{2}$ satisfying 


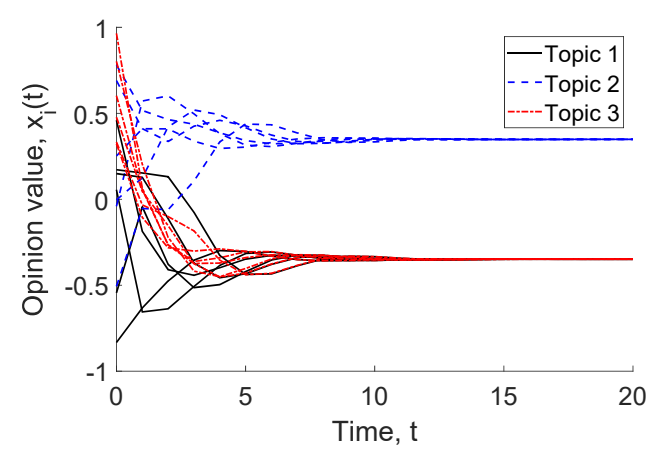

Figure 3. Evolution of opinions for 3 topics, with $\boldsymbol{C}_{i}$ given in Eq. (16).

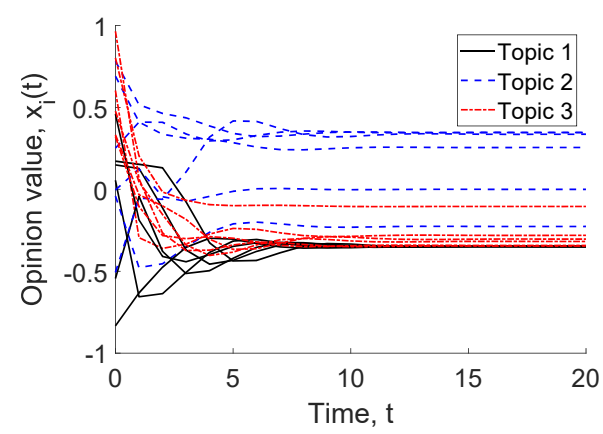

Figure 4. Evolution of opinions for 3 topics, with $\boldsymbol{C}_{i}$ given in Eq. (16), except $\boldsymbol{C}_{1}$ has entry $c_{21,1}=\beta_{1}$.

Eq. (13), and Theorem 3 predicts that the final opinions of Topic 2 will be at a disagreement. This is also established in Corollary 2, Item 1), and is clearly observed in Fig. 4. Because Topic 3 is dependent on Topic 2, one observes in Fig. 4 that Topic 3 also fails to reach a consensus (see the conjecture in Remark 3 in Section IV-B below).

Example 2: Now, we illustrate Theorem 4 and Eq. (15) with an example of 3 topics, i.e. $\mathcal{J}=\{1,2,3\}$. For all $i=1, \ldots, 6$, the logic matrix is

$$
\boldsymbol{C}_{i}=\left[\begin{array}{ccc}
1 & 0 & 0 \\
\theta \beta_{i} & 1-(1+\theta) \beta_{i} & -\beta_{i} \\
\tau \delta_{i} & -\delta_{i} & 1-\delta_{i}(1+\tau)
\end{array}\right]
$$

where $\theta, \tau$ are positive scalars and $\beta_{i}$ and $\delta_{i}$ are randomly drawn from a uniform distribution in the interval $(0,1)$, and appropriately normalised. In our particular example, we choose $\theta=0.5$ and $\tau=0.3$. According to the notation of Eq. (10), we have $\mathcal{J}_{1}=\{1\}$ (closed), $\mathcal{J}_{2}=\{2,3\}$ (open), and the temporal evolution of $\boldsymbol{x}(t)$ is given in Fig. 5 .

Consistent with Theorem 2, Topic 1 reaches a consensus, with a value in this example of $\alpha_{1}=0.655$. We consider the open strongly connected component comprising of Topics 2 and 3, for which Theorem 4 is applicable. Following Theorem 4's notation, $j=2$, so that $\mathcal{J}_{2}=\{2,3\}$ and $\tilde{\mathcal{J}}_{2}=\{1\}$. Define $\boldsymbol{\beta}$ and $\boldsymbol{\Delta}$ to be $n \times n$ diagonal matrices with $i^{\text {th }}$ entry being $\beta_{i}$ and $\delta_{i}$, respectively. Eq. (15) evaluates to be

$$
\left[\begin{array}{cc}
(1+\theta) \boldsymbol{\beta} & \boldsymbol{\beta} \\
\boldsymbol{\Delta} & (1+\tau) \boldsymbol{\Delta}
\end{array}\right]\left(\left[\begin{array}{l}
\phi_{2} \\
\phi_{3}
\end{array}\right] \otimes \mathbf{1}_{n}\right)=\alpha_{1}\left[\begin{array}{c}
\theta \boldsymbol{\beta} \\
\tau \boldsymbol{\Delta}
\end{array}\right] \mathbf{1}_{2 n}
$$

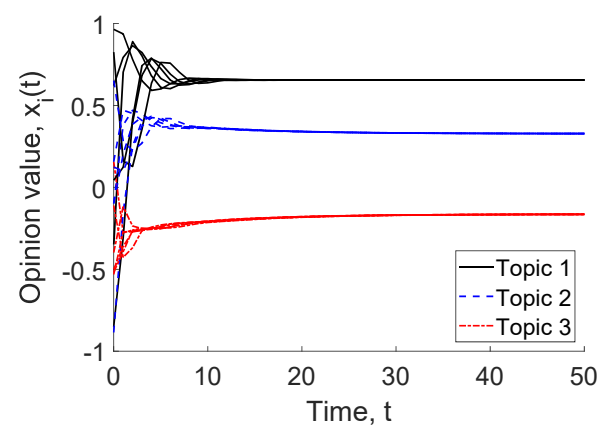

Figure 5. Evolution of opinions for 3 topics, with $\boldsymbol{C}_{i}$ given in Eq. (17).

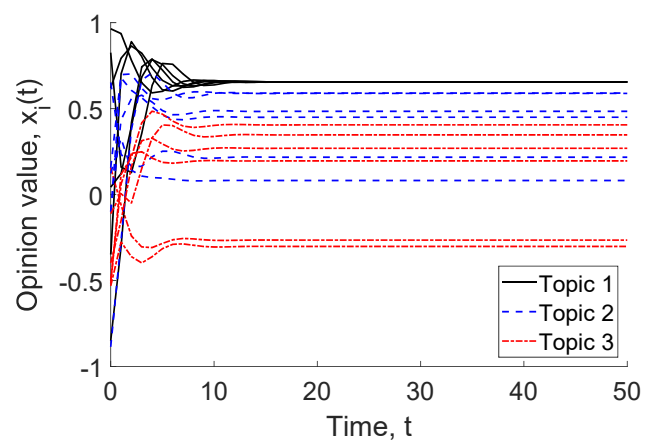

Figure 6. Evolution of opinions for 3 topics, with $\boldsymbol{C}_{i}$ given in Eq. (19).

Substituting in $\alpha_{1}=0.655, \theta=0.5$ and $\tau=0.2$, one can verify that Eq. (18) holds for $\phi_{2}=0.3275$ and $\phi_{3}=-0.1637$. Theorem 4 predicts that Topics 2 and 3 will reach a consensus, with values $\phi_{2}$ and $\phi_{3}$, respectively. Indeed, this is observed in Fig. 5. It is important to note that although $\alpha_{1}$ depends on $\boldsymbol{x}(0)$, there exists a $\boldsymbol{\phi}$ satisfying Eq. (15) for all $\alpha_{1} \in[-1,1]$. In other words, the consensus of Topics 2 and 3 are robust to variations in $\boldsymbol{x}(0)$, and also robust to values of parameters $\theta, \tau$ (with appropriate normalisation).

The entries of $\boldsymbol{C}_{i}$ in Eq. (17) have a specific relationship: $c_{21, i}$ and $c_{31, i}$ are scalar multiples of $c_{23, i}$ and $c_{32, i}$, respectively, with the scalars independent of $i$. Suppose the scalar relationships did not hold, and instead the logic matrix was

$$
\boldsymbol{C}_{i}=\left[\begin{array}{ccc}
1 & 0 & 0 \\
\eta_{i} & 1-\left(\beta_{i}+\eta_{i}\right) & -\beta_{i} \\
\mu_{i} & -\delta_{i} & 1-\left(\delta_{i}+\mu_{i}\right)
\end{array}\right],
$$

where $\eta_{i}, \beta_{i}, \mu_{i}, \delta_{i}$ are independently randomly drawn from a uniform distribution in the interval $(0,1)$ with appropriate normalisation. Generically, there will not exist $\phi$ satisfying Eq. (15), and so a consensus will not be reached; an example simulation is presented in Fig. 6. From numerous simulations, we observed that Eq. (15) is generally much harder to satisfy than Eq. (13), indicating open strongly connected components of two or more topics are less likely to reach a consensus.

\section{B. Discussion and Social Interpretations}

We now provide some discussion and comments on the main results, focusing in particular on the theorems and corollaries 
in Section III-B. Overall, the outcomes we have established depend on the graphical structures $\mathcal{G}\left[\boldsymbol{C}_{i}\right]$ on the one hand, and on the numerical values (including their signs) of the $\boldsymbol{C}_{i}$ entries on the other.

This dependence sometimes flows simply from the signs (the presence or absence of competing logical interdependencies), such as Example 1 in Section IV-A. At other times, the precise values of the $\boldsymbol{C}_{i}$ matter, as illustrated in Example 2 of Section IV-A (compare Eq. (17) and Eq. (19)). Further, when consensus on a topic occurs, it is evident that sometimes a value 0 is always the outcome, and sometimes a nonzero value dependent on the initial opinions of topics in the closed strongly connected components of $\mathcal{G}\left[\boldsymbol{C}_{i}\right]$. Moreover, the consensus point for each topic can differ in both sign and absolute value, as in Example 2 of Section IV-A, Fig. 5.

One interpretation of a topic set $\mathcal{J}_{j}$ corresponding to a closed strongly connected component of $\mathcal{G}\left[\boldsymbol{C}_{i}\right]$ is that the topic(s) is an axiom(s) upon which an individual builds his or her belief system (see below Eq. (10)). Theorem 2 indicates that discussion of axiomatic topics will always lead a consensus under the model Eq. (1).

Theorem 2 and Corollaries 2 and 3 also illustrate that competing logical interdependencies, if present, can play a major role in determining the final opinion values. For a topic set $\mathcal{J}_{j}$ corresponding to a closed and strongly connected component of $\mathcal{G}\left[\boldsymbol{C}_{i}\right]$, all opinion values for all topics in $\mathcal{J}_{j}$ converge to the neutral value at 0 whenever competing interdependencies are present in $\mathcal{J}_{j}$ (Theorem 2, Item 2)). For an open strongly connected component $\mathcal{J}_{j}$, the presence of any competing logical interdependencies in topic $p \in \mathcal{J}_{j}$ is enough to prevent the sufficient conditions detailed in Corollary 2 Item $1), 3$ ), and 4) and Corollary 3 Item 2) from being satisfied. Of particular note is Corollary 2 Item 1). As illustrated in Example 1 in Section IV-A, heterogeneity with respect to $i$ in the entries of $c_{21, i}$ is not enough to prevent a consensus of opinions on Topic 2; competing logical interdependences are required. The necessity of the competing logical interdependencies for disagreement is a surprising, and non-intuitive result.

It is also clear from Theorems 2, 3 and 4 that disagreement is possible only in topic sets $\mathcal{J}_{j}$ associated with an open strongly connected component of $\mathcal{G}\left[\boldsymbol{C}_{i}\right]$. Put another way, belief systems with a cascade logical structure, viz. reducible $\boldsymbol{C}_{i}$ in the form of Eq. (9), including heterogeneity among individuals' belief systems, may play a significant role in generating disagreement when social networks discuss multiple logically interdependent topics. Looking at Eq. (1), one can see two separate processes occurring: the DeGroot component describes interpersonal influence between individuals in an effort to reach a consensus, while the logic matrix by itself (as in Eq. (3)) captures an intrapersonal effort to secure logical consistency of opinions across several topics. These two drivers may or may not end up in conflict, and the presence of conflict or lack thereof determines whether opinions of a certain topic reach a consensus or fail to do so. Our results in Theorems 3 and 4 identify when such conflict can occur.

Remark 3. Theorems 3 and 4 establish necessary and sufficient conditions for topic $j_{k} \in \mathcal{J}_{j}=\left\{j_{1}, \ldots, j_{z}\right\}$ to reach a consensus under a particular hypothesis. Specifically, it is assumed that for the set $\mathcal{J}_{j}$ under consideration, there holds

$$
\boldsymbol{y}_{q}^{*}=\alpha_{q} \mathbf{1}_{n}, \quad \forall q \in \tilde{\mathcal{J}}_{j} .
$$

That is, all other topics that one or more topics $j_{k} \in \mathcal{J}_{j}$ depend upon are assumed to have reached a consensus. Based on numerous simulations, we believe the requirement that Eq. (20) holds is also a necessary condition for $\boldsymbol{y}_{j_{k}}, j_{k} \in \mathcal{J}_{j}$ to reach a consensus. In other words, if any topic $q \in \tilde{\mathcal{J}}_{j}$ fails to reach a consensus, we conjecture that all $\boldsymbol{y}_{j_{k}}, k=1, \ldots, z$ will also fail to reach a consensus. Confirming this would provide yet another indication that networks with belief systems having a cascade logic structure more readily result in disagreement. We leave this to future investigations.

\section{Novel Insight Into Strong Diversity}

We now compare our findings with those in the existing literature. The dynamics Eq. (1) is a particular variation on the model studied in [18], [19], and we explain why our findings are especially illuminating.

For any $\boldsymbol{W}$ satisfying Assumption 3, it is known that $\lim _{k \rightarrow \infty} \boldsymbol{W}^{k}=\mathbf{1}_{n} \boldsymbol{\gamma}^{\top}$ where $\boldsymbol{\gamma}^{\top}$ is a left eigenvector of $\boldsymbol{W}$ associated with the simple eigenvalue at 1 , having entries $\gamma_{j}>0$, and normalised to satisfy $\gamma^{\top} \mathbf{1}_{n}=1$ [26]. Supposing that the logic matrices were indeed homogeneous, i.e. $C_{i}=C_{j}=C$ for all $i, j \in \mathcal{I}$, we can verify that much of the analysis becomes easier. For then Eq. (6) becomes $\boldsymbol{x}(t+1)=(\boldsymbol{W} \otimes \boldsymbol{C}) \boldsymbol{x}(t)$, and the limiting behaviour is characterised by the following result (which is a restatement of [18, Theorem 3]).

Theorem 5. Suppose Assumptions 1, 2, and 3 hold. Suppose further that $\boldsymbol{C}_{i}=\boldsymbol{C}_{j}=\boldsymbol{C}$ for all $i, j \in \mathcal{I}$. Then, the system Eq. (6) converges as $\lim _{t \rightarrow \infty} \boldsymbol{x}_{i}(t)=\sum_{j=1}^{n} \gamma_{j} \boldsymbol{C}^{t} \boldsymbol{x}_{j}(0)$, where $\gamma_{j}>0$ is detailed immediately above.

Theorem 5 enables us to compare the results between Theorems 2, 3, and 4 of this paper with the case in [18] where $C$ is homogeneous (under the same Assumptions 1, 2, and 3). Theorem 5 shows that if $C$ is homogeneous, then the opinions of all individuals on any given topic reach a consensus: Eq. (4) is satisfied for all $k \in \mathcal{J}$.

The Friedkin-Johnsen variant to Eq. (1) is also studied in [18], [19], and is given as

$$
\boldsymbol{x}_{i}(t+1)=\lambda_{i} \sum_{j=1}^{n} w_{i j} \boldsymbol{C}_{i} \boldsymbol{x}_{j}(t)+\left(1-\lambda_{i}\right) \boldsymbol{x}_{i}(0) .
$$

Here, the parameter $\lambda_{i} \in[0,1]$ represents individual $i$ 's susceptibility to interpersonal influence, while $1-\lambda_{i}$ represents the level of stubborn attachment ${ }^{3}$ by individual $i$ to his/her initial opinions $\boldsymbol{x}_{i}(0)$. This paper studies the special case where $\lambda_{i}=1, \forall i \in \mathcal{I}$, and thus Eq. (21) and Eq. (1) are equivalent. When $\boldsymbol{W}$ satisfies Assumption 3 and $\exists i, j \in \mathcal{I}$ with $i \neq j$ such that $\lambda_{i}, \lambda_{j}<1$, a strong diversity of opinions

\footnotetext{
${ }^{3}$ The paper [19] secures a convergence result for heterogeneous $\boldsymbol{C}_{i}$ but makes an assumption that there is at least one individual $i$ with $\lambda_{i}<1$. [18] assumes homogeneous $\boldsymbol{C}$.
} 
emerges if $\boldsymbol{x}_{i}(0) \neq \boldsymbol{x}_{j}(0)$ as a consequence of individuals $i$ and $j$ having some level of attachment to their initial opinions $\boldsymbol{x}_{i}(0), \boldsymbol{x}_{j}(0)$, even if $\boldsymbol{C}_{i}=\boldsymbol{I}_{m}$ for all $i \in \mathcal{I}$ [18], [19].

In contrast, this paper has assumed heterogeneous $\boldsymbol{C}_{i}$ and $\lambda_{i}=0$ for all $i$, and shown that opinions on a given topic can fail to reach a consensus with instead strong diversity emerging. Specifically, Theorem 2 shows that when the $\boldsymbol{C}_{i}$ are irreducible, a consensus is achieved for each topic, and is thus consistent with the results observed in [18]. However, different phenomena are generated when $C_{i}$ are reducible, viz. consensus of each topic for homogeneous $C$ (as in [18]) and disagreement for heterogeneous $\boldsymbol{C}_{i}$ (Theorems 3 and 4). Consequently, we have isolated and highlighted the separate roles of both the structure and the heterogeneity of the $C_{i}$ among individuals, in creating strong diversity. In fact, a cascade logic structure is necessary for disagreement. This constitutes a novel insight into the emergence of strong diversity in strongly connected networks, linking it for the first time to differences in individuals' belief systems as opposed to stubbornness [13], a desire to be unique [23], [24], or social distancing [23].

\section{Conclusion}

We have studied influence networks in which individuals discuss a set of logically interdependent topics, assuming that the network has no stubborn individuals in order to focus on the effects of the logical interdependence structure. We established that for strongly connected networks, and reasonable assumptions on the logic matrix, the opinions converge exponentially fast to some steady-state value. We then provided a systematic way to help determine whether a given topic will reach a consensus or fail to do so. It was discovered that heterogeneity of reducible logic matrices among individuals, including differences in signs of the off-diagonal entries, played a primary role in producing disagreement in the final opinion values. In the problem context, we have established that a cascade logic structure and heterogeneity of individuals' belief systems are necessary to generate the phenomenon of strong diversity of final opinions. Competing logical interdependencies can also play an important role. We believe these are key new insights and explanation of strong diversity, as most existing works attribute strong diversity in connected networks to factors such as individual stubbornness. Future work will focus on proving the conjecture in Remark 3, relaxing Assumption 2, asynchronous updating (e.g. gossiping as studied in [18]), and the effect of the logic matrix on the convergence rate. State-dependent connectivity in the interpersonal interactions or logical interdependencies is also of interest, for which a hybrid framework may be relevant [30].

\section{APPENDIX}

We give some definitions and results to be used in the analysis. The infinity norm and spectral radius of a square matrix $\boldsymbol{A}$ are denoted by $\|\boldsymbol{A}\|_{\infty}$ and $\rho(\boldsymbol{A})$, respectively. For a matrix $\boldsymbol{A}$, let $|\boldsymbol{A}|$ be the matrix whose $i j^{\text {th }}$ entry is the absolute value of the $i j^{t h}$ entry of $\boldsymbol{A}$. A square $\boldsymbol{A} \geq 0$ is primitive if $\exists k \in \mathbb{N}: \boldsymbol{A}^{k}>0$ [26, Definition 1.12]. For some $\boldsymbol{A} \geq 0$, the graph $\mathcal{G}[\boldsymbol{A}]$ is strongly connected and aperiodic if and only if $\boldsymbol{A}$ is primitive [26, Proposition 1.35]. The irreducibility of $\boldsymbol{A}$ (equivalent to strong connectivity of $\mathcal{G}[\boldsymbol{A}]$ ) implies that if a $k$ exists such that $\boldsymbol{A}^{k}>0$, then $\boldsymbol{A}^{j}>0$ for all $j>k$. Last, we establish the following helpful lemma.

Lemma 1. Suppose that Assumption 2 holds. Then, $\mathcal{G}[\boldsymbol{A}]$, where $\boldsymbol{A}$ is the matrix in Eq. (8), is strongly connected and aperiodic if and only if, separately, $\mathcal{G}[\boldsymbol{W}]$ and $\mathcal{G}\left[\boldsymbol{C}_{i}\right], \forall i$ are strongly connected and aperiodic.

Proof. Let $\bar{C}$ be a nonnegative row-stochastic matrix with the same zero and non-zero pattern of entries as $C_{i}, \forall i \in \mathcal{I}$, i.e. $\overline{\boldsymbol{C}} \sim \boldsymbol{C}_{i}, \forall i \in \mathcal{I}$. Then, by the lemma hypothesis on Assumption 2, the graph $\mathcal{G}[\overline{\boldsymbol{C}} \otimes \boldsymbol{W}]$ has the same vertex and edge set as $\mathcal{G}[\boldsymbol{A}]$, but with different edge weights (including the fact that all edge weights of $\mathcal{G}[\overrightarrow{\boldsymbol{C}} \otimes \boldsymbol{W}]$ are positive, whereas negative edge weights may exist in $\mathcal{G}[\boldsymbol{A}]$ ). One can prove that $\mathcal{G}[\overline{\boldsymbol{C}} \otimes \boldsymbol{W}]$ is strongly connected and aperiodic by using [31, Theorem 1], and this is equivalent to proving that $\mathcal{G}[\boldsymbol{A}]$ is strongly connected and aperiodic.

\section{A. Theorem 1}

The proof has two parts: in Part 1 and Part 2, we prove convergence for irreducible and reducible $C_{i}$, respectively.

Part 1: Consider the case where all the $\boldsymbol{C}_{i}$ are irreducible (i.e. $\mathcal{G}\left[\boldsymbol{C}_{i}\right]$ is strongly connected). We have that $\mathcal{G}[\boldsymbol{W}]$ and $\mathcal{G}\left[\boldsymbol{C}_{i}\right], \forall i \in \mathcal{I}$ are separately strongly connected and aperiodic from Assumptions 1, 2, and 3 (the aperiodicity is a consequence of the assumption that $w_{i i}>0$ and $c_{p p, i}>0$ for all $i \in \mathcal{I}$ and $p \in \mathcal{J}$ ). From [26, Proposition 1.35], we then conclude that $\mathcal{G}[\boldsymbol{A}]$ is strongly connected and aperiodic. Moreover, every diagonal entry of $\boldsymbol{A}$ is strictly positive. Using existing results on the Altafini model for strongly connected networks [10, Theorem 1 and 2], we conclude that $\lim _{t \rightarrow \infty} \boldsymbol{y}(t)=\boldsymbol{y}^{*}$ exponentially fast, where $\boldsymbol{y}^{*} \in \mathbb{R}^{n m}$ is the steady-state opinion distribution.

Part 2: Consider now the case where all $\boldsymbol{C}_{i}$ are reducible, with $\boldsymbol{C}_{i}$ having the form in Eq. (9), $\mathcal{S} \triangleq\{1,2, \ldots s\}$, and $s_{j}$ being integers satisfying $\sum_{j=1}^{s} s_{j}=m$. The matrix $\boldsymbol{A}$ in Eq. (8) has the following form

$$
\boldsymbol{A}=\left[\begin{array}{cccc}
\overline{\boldsymbol{A}}_{11} & \mathbf{0} & \cdots & \mathbf{0} \\
\overline{\boldsymbol{A}}_{21} & \overline{\boldsymbol{A}}_{22} & \cdots & \mathbf{0} \\
\vdots & \vdots & \ddots & \vdots \\
\overline{\boldsymbol{A}}_{s 1} & \overline{\boldsymbol{A}}_{s 2} & \cdots & \overline{\boldsymbol{A}}_{s s}
\end{array}\right]
$$

with block matrix elements $\bar{A}_{p q}, p, q \in \mathcal{S}$ given

$$
\overline{\boldsymbol{A}}_{p q}=\left[\begin{array}{cccc}
\boldsymbol{A}_{g h} & \boldsymbol{A}_{g, h+1} & \cdots & \boldsymbol{A}_{g, h+s_{q}-1} \\
\boldsymbol{A}_{g+1, h} & \boldsymbol{A}_{g+1, h+1} & \cdots & \boldsymbol{A}_{g+1, h+s_{q}-1} \\
\vdots & \vdots & \ddots & \vdots \\
\boldsymbol{A}_{g+s_{p}-1, h} & \boldsymbol{A}_{g+s_{p}-1, h+1} & \cdots & \boldsymbol{A}_{g+s_{p}-1, h+s_{q}-1} \cdot
\end{array}\right]
$$

Here, $g=\sum_{i=1}^{p} s_{i-1}+1$ and $h=\sum_{i=1}^{q} s_{i-1}+1$ for $p, q \in \mathcal{S}$ with $s_{0}=0$. From the decomposition in Eq. (9), we know that $C_{p p, i}$ is irreducible for any $p \in \mathcal{S}$ and $i \in \mathcal{I}$, and all the diagonal entries are positive (see Assumption 1). This implies that $\mathcal{G}\left[\boldsymbol{C}_{p p, i}\right]$ is strongly connected and apediodic. 
We prove the exponential convergence property by induction. First, for the base case consider the topics in $\mathcal{J}_{1}$, which are $\left\{1,2, \ldots, s_{1}\right\}$. Since $\boldsymbol{C}_{11, i}$ is irreducible for all $i \in \mathcal{I}$, we obtain from Part 1 that for all topics $k \in \mathcal{J}_{1}$, there holds $\lim _{t \rightarrow \infty} \boldsymbol{y}_{k}(t)=\boldsymbol{y}_{k}^{*}$ exponentially fast, for some $\boldsymbol{y}_{k}^{*} \in \mathbb{R}^{n}$.

We now prove the induction step for topic $k$ in the topic subset $\mathcal{J}_{p}$, with $p \in \mathcal{S}$ and $p \geq 2$. Suppose that for all topics $l \in \cup_{j=1}^{p-1} \mathcal{J}_{j}, \lim _{t \rightarrow \infty} \boldsymbol{y}_{l}(t)=\boldsymbol{y}_{l}^{*}$ exponentially fast, where $\boldsymbol{y}_{l}^{*}$ is the vector of final opinions. We need to show that for all topics $k$ in $\mathcal{J}_{p}$, there exists a vector $\boldsymbol{y}_{k}^{*} \in \mathbb{R}^{n}$ such that there holds $\lim _{t \rightarrow \infty} \boldsymbol{y}_{k}(t)=\boldsymbol{y}_{k}^{*}$ exponentially fast. Look at the $p$-th block row of matrix $\boldsymbol{A}$. Suppose first that $\overline{\boldsymbol{A}}_{p q}=\mathbf{0}$ for $q<p$. Since $C_{p p, i}$ is irreducible for any $i \in \mathcal{I}$, then by the analysis in Part 1 of this proof, we conclude that for every $k \in \mathcal{J}_{p}$, there exists a $\boldsymbol{y}_{k}^{*} \in \mathbb{R}^{n}$ such that $\lim _{t \rightarrow \infty} \boldsymbol{y}_{k}(t)=\boldsymbol{y}_{k}^{*}$ exponentially fast. Next, suppose to the contrary, that there exists a $q<p$ such that $\overline{\boldsymbol{A}}_{p q} \neq \mathbf{0}$. Because $\mathcal{G}\left[\boldsymbol{C}_{p p, i}\right], \forall i \in \mathcal{I}$ are strongly connected and aperiodic, one can apply Lemma 1 to obtain that $\mathcal{G}\left[\overline{\boldsymbol{A}}_{p p}\right]$ is strongly connected and aperiodic, i.e. $\overline{\boldsymbol{A}}_{p p}$ is irreducible. Since $\overline{\boldsymbol{A}}_{p p}$ is irreducible, $\left|\overline{\boldsymbol{A}}_{p p}\right|$ is also irreducible. Because $\exists q<p$ such that $\overline{\boldsymbol{A}}_{p q} \neq \mathbf{0}$, we conclude that $\left|\overline{\boldsymbol{A}}_{p p}\right|$ is row-substochastic. It follows from [32, Lemma 2.8] that $\rho\left(\left|\overline{\boldsymbol{A}}_{p p}\right|\right)<1$. Using the triangle inequality, verify that the $i j^{t h}$ entry of $\left|\overline{\boldsymbol{A}}_{p p}^{k}\right|$ is less than or equal to the $i j^{t h}$ entry of $\left|\bar{A}_{p p}\right|^{k}$. Thus

$$
\left\|\overline{\boldsymbol{A}}_{p p}^{k}\right\|_{\infty}=\left\|\left|\overline{\boldsymbol{A}}_{p p}^{k}\right|\right\|_{\infty} \leq\left\|\left|\overline{\boldsymbol{A}}_{p p}\right|^{k}\right\|_{\infty} .
$$

It follows that $\lim _{k \rightarrow \infty}\left\|\overline{\boldsymbol{A}}_{p p}^{k}\right\|_{\infty}^{1 / k} \leq \lim _{k \rightarrow \infty}\left\|\left|\overline{\boldsymbol{A}}_{p p}\right|^{k}\right\|_{\infty}^{1 / k}$, which in turn implies that $\rho\left(\overline{\boldsymbol{A}}_{p p}\right) \leq \rho\left(\left|\overline{\boldsymbol{A}}_{p p}\right|\right)<1$. Recall that at the start of the induction step, we assumed that for all $l \in \cup_{j=1}^{p-1} \mathcal{J}_{j}$ (with $p \in \mathcal{S}$ and $p \geq 2$ ), there exists $\boldsymbol{y}_{l}^{*} \in \mathbb{R}^{n}$ such that $\lim _{t \rightarrow \infty} \boldsymbol{y}_{l}(t)=\boldsymbol{y}_{l}^{*}$ exponentially fast. Combining this assumption with the fact that $\rho\left(\overline{\boldsymbol{A}}_{p p}\right)<1$, we conclude that for every $k \in \mathcal{J}_{p}$, there exists a $\boldsymbol{y}_{k}^{*} \in \mathbb{R}^{n}$ such that $\lim _{t \rightarrow \infty} \boldsymbol{y}_{k}(t)=\boldsymbol{y}_{k}^{*}$ exponentially fast.

The invariance property in which $y_{i}^{p}(0) \in[-1,1]$ for all $i \in \mathcal{I}$ and $p \in \mathcal{J}$ guarantees $y_{i}^{p}(t) \in[-1,1]$ for all $t \geq 0$ and $i \in \mathcal{I}$ and $p \in \mathcal{J}$ was proved in [18]. It relies on the fact that $\sum_{q=1}^{n}\left|c_{p q, i}\right|=1$ as detailed in Assumption 1.

\section{B. Analysis for Subsection III-B}

Here, we present a supporting result that links the structural balance of the graph $\mathcal{G}[\boldsymbol{A}]$ to the structural balance of $\mathcal{G}\left[\boldsymbol{C}_{i}\right], i \in \mathcal{I}$, which will be used to help prove the main result on consensus for irreducible $\boldsymbol{C}_{i}$.

First, we introduce additional graph-theoretic concepts. For a given (possibly signed) graph $\mathcal{G}$, an undirected cycle is a cycle of $\mathcal{G}$ that ignores the direction of the edges, and an undirected cycle is negative if it contains an odd number of edges with negative edge weight. A signed graph $\mathcal{G}$ is structurally unbalanced if and only if it has at least one negative undirected cycle [27].

We now establish several additional properties of how the entries $c_{i j, k}$ of $\boldsymbol{C}_{k}$ relate to edges in $\mathcal{G}[\boldsymbol{A}]$.

Lemma 2. For the graph $\mathcal{G}[\boldsymbol{A}]$ with node set $\mathcal{V}[\boldsymbol{A}]=$ $\left\{v_{1}, \ldots, v_{n m}\right\}$, let $\mathcal{V}_{p}=\left\{v_{(p-1) n+1}, \ldots, v_{p n}\right\}, p \in \mathcal{J}$ be defined as the set of nodes of the subgraph $\mathcal{G}\left[\boldsymbol{A}_{p p}\right]$. Suppose that Assumptions 1, 2 and 3 hold. Then,

1) For every $p \in \mathcal{J}, \mathcal{G}\left[\boldsymbol{A}_{p p}\right]$ is strongly connected and aperiodic with positive edge weights.

2) There is an edge from node $v_{(q-1) n+j}$ to $v_{(p-1) n+i}$ if and only if $w_{i j}>0$ and $c_{p q, i} \neq 0$. Moreover, the weight of the edge the same sign as the sign of $c_{p q, i}$.

3) If $c_{p q, k} \neq 0 \forall k \in \mathcal{I}$, then with $p \neq q$, every node in $\mathcal{V}_{p}$ has an incoming edge from a node $\mathcal{V}_{q}$, and every node in $\mathcal{V}_{q}$ has an outgoing edge to a node in $\mathcal{V}_{p}$.

Proof. First, recall that $\boldsymbol{A}_{p q} \triangleq \boldsymbol{\Gamma}_{p q} \boldsymbol{W}$ as below Eq. (7).

Item 1): From Assumption 1, we know that $c_{p p, i}>0 \forall i \in \mathcal{I}$ and $p \in \mathcal{J}$. This implies that $\boldsymbol{A}_{p p} \sim \boldsymbol{W}$ and $\boldsymbol{A}_{p p} \geq 0$ with all positive diagonals, which implies that $\mathcal{G}\left[\boldsymbol{A}_{p p}\right]$ is strongly connected and aperiodic.

Item 2): Notice that $\boldsymbol{A}_{p q}$ is nonzero if and only if $c_{p q, i} \neq$ $0, i \in \mathcal{I}$. Moreover, the $i j^{t h}$ entry of a nonzero $\boldsymbol{A}_{p q}$ is nonzero if and only if $w_{i j}>0$, and has the same sign as $c_{p q, i}$. Recall that we defined node subsets $\mathcal{V}_{p}=\left\{v_{(p-1) n+1}, \ldots, v_{p n}\right\}$, $p \in \mathcal{J}$ for the graph $\mathcal{G}[\boldsymbol{A}]$. It follows that an edge from node $v_{(q-1) n+j} \in \mathcal{V}_{q}$ to $v_{(p-1) n+i} \in \mathcal{V}_{p}$ exists if and only if $w_{i j}>0$ and $c_{p q, i} \neq 0$, and has the same sign as $c_{p q, i}$.

Item 3): This statement is obtained by (i) recalling the definition of the node set $\mathcal{V}_{p}=\left\{v_{(p-1) n+1}, \ldots, v_{p n}\right\}, p \in \mathcal{J}$, (ii) observing that an irreducible $\boldsymbol{W}$ implies that for any $i \in \mathcal{I}$, there exists a $j \in \mathcal{I}, i \neq j$ such that $w_{i j}>0$, and (iii) by applying Item 2).

We now turn to study of the structural balance of $\mathcal{G}[\boldsymbol{A}]$ and its relation to the structural balance of the $\mathcal{G}\left[\boldsymbol{C}_{i}\right] \mathrm{s}$.

Lemma 3. Suppose that Assumptions 1, 2, and 3 hold. Suppose further that $\boldsymbol{C}_{i}$ for all $i \in \mathcal{I}$ are irreducible. The following hold:

1) If there are no individuals with competing logical interdependencies, as given in Definition 1 , then $\mathcal{G}[\boldsymbol{A}]$ is structurally balanced if and only if $\mathcal{G}\left[\boldsymbol{C}_{i}\right], \forall i \in \mathcal{I}$ are structurally balanced.

2) If there are individuals with competing logical interdependences, then $\mathcal{G}[\boldsymbol{A}]$ is structurally unbalanced.

Proof. We prove each statement separately.

Part 1: Consider the case where there are no individuals with competing logical interdependencies. Since, for any $p, q \in \mathcal{J}, c_{p q, i}$ for all $i \in \mathcal{I}$ are of the same sign, it follows that all graphs $\mathcal{G}\left[\boldsymbol{C}_{i}\right]$ have the same structural balance or unbalance property. Moreover, because the structural balance or unbalance property of any graph depends on the sign, and not the magnitude, of its edge weights, let us consider $\mathcal{G}\left[\boldsymbol{C}_{1}\right]$ for convenience. For brevity, we also drop the subscript 1 and simply write $\mathcal{G}[\boldsymbol{C}]$ for Part 1 of this proof, with node set $\mathcal{V}_{\boldsymbol{C}}=\left\{v_{c, 1}, \ldots, v_{c, m}\right\}$. To establish the result, we will exploit Lemma 2. For each $p \in \mathcal{J}$, consider the subgraph $\mathcal{G}\left[\boldsymbol{A}_{p p}\right]$ of $\mathcal{G}[\boldsymbol{A}]$. Item 1) of Lemma 2 tells us that every edge in $\mathcal{G}\left[\boldsymbol{A}_{p p}\right]$ has a positive weight, while Item 2) and Item 3) of Lemma 2 establish that the edge weights for all edges from $\mathcal{G}\left[\boldsymbol{A}_{q q}\right]$ to $\mathcal{G}\left[\boldsymbol{A}_{p p}\right]$ have the same sign as the sign of the weight for the edge $\left(v_{c, q}, v_{c, p}\right)$ in $\mathcal{G}[\boldsymbol{C}]$. 
With these properties in mind, consider a structurally unbalanced $\mathcal{G}[\boldsymbol{C}]$; since $\mathcal{G}[\boldsymbol{C}]$ is strongly connected, the unbalance property implies there is at least one negative directed cycle. Without loss of generality, consider the negative cycle

$$
\left(v_{c, p}, v_{c, z_{1}}\right),\left(v_{c, z_{1}}, v_{c, z_{2}}\right), \ldots\left(v_{c, z_{r}}, v_{c, p}\right)
$$

with $z_{1}, \ldots z_{r} \in \mathcal{J}$ and $r \geq 1$. Let $u \in \mathbb{N}$ be the odd number of negative edges in the undirected cycle. From Item 2) and 3) of Lemma 2 , and using the fact that $w_{i i}>0 \forall i \in \mathcal{I}$, we conclude that $\mathcal{G}[\boldsymbol{A}]$ has an undirected cycle

$$
\begin{aligned}
\pi= & \left(v_{(p-1) n+i}, v_{\left(z_{1}-1\right) n+i}\right),\left(v_{\left(z_{1}-1\right) n+i}, v_{\left(z_{2}-1\right) n+i}\right), \ldots, \\
& \left(v_{\left(z_{r}\right) n+i}, v_{(p-1) n+i}\right) .
\end{aligned}
$$

The undirected cycle $\pi$ contains precisely $u$ edges with negative weight, which implies that $\pi$ is a negative cycle. It follows that $\mathcal{G}[\boldsymbol{A}]$ is structurally unbalanced.

Next, consider a structurally balanced $\mathcal{G}[\boldsymbol{C}]$, and assume without loss of generality that the nodes are ordered such that they can be partitioned into disjoint sets $\mathcal{V}^{+}=\left\{v_{c, 1}, \ldots, v_{c, s}\right\}$ and $\mathcal{V}^{-}=\left\{v_{c, s+1}, \ldots, v_{c, m}\right\}$, with $1 \leq s<m$. The two sets have the property that each edge between two nodes in $\mathcal{V}^{+}$or $\mathcal{V}^{-}$has positive weight, while each edge between a node in $\mathcal{V}^{+}$and a node in $\mathcal{V}^{-}$has negative weight. Without loss of generality, consider an undirected cycle, $\pi$, in $\mathcal{G}[\boldsymbol{A}]$ starting and ending at a node $\bar{v}$ in the subgraph $\mathcal{G}\left[\boldsymbol{A}_{11}\right]$. We are going to show that any such $\pi$ is not a negative undirected cycle. If $\pi$ traverses only nodes in $\mathcal{G}\left[\boldsymbol{A}_{11}\right]$, then clearly all edges on the path have positive weight. Suppose instead that $\pi$ is such that it traverses at least one node in each of the subgraphs $\mathcal{G}\left[\boldsymbol{A}_{11}\right]$, $\mathcal{G}\left[\boldsymbol{A}_{z_{1} z_{1}}\right], \ldots, \mathcal{G}\left[\boldsymbol{A}_{z_{r} z_{r}}\right]$, with $z_{1}, \ldots z_{r} \in \mathcal{J}$ and $r \geq 1$ (by the definition of an undirected cycle, each node in the cycle apart from $\bar{v}$ is distinct). If $v_{c, z_{1}}, \ldots, v_{c, z_{r}} \in \mathcal{V}^{+}$, then we conclude from Item 2) and 3) of Lemma 2 that all edges in $\pi$ have positive weight. In both cases, $\pi$ is not a negative undirected cycle. Now suppose that $z_{1}, \ldots z_{k}$, with $k<r$, are such that $v_{c, z_{1}}, \ldots v_{c, z_{k}} \in \mathcal{V}^{-}$. Notice that for any two nodes $\tilde{v}$ and $\hat{v}$ in the subgraphs $\mathcal{G}\left[\boldsymbol{A}_{p p}\right], p \in\{1, \ldots, s\}$, a path from $\tilde{v}$ to $\hat{v}$ which traverses nodes in the subgraphs $\mathcal{G}\left[\boldsymbol{A}_{q q}\right], q \in\{s+$ $1, \ldots, m\}$ has an even number of edges with negative weight. This is because $v_{c, p} \in \mathcal{V}^{+}, p \in\{1, \ldots, s\}$ and $v_{c, q} \in \mathcal{V}^{-}, q \in$ $\{s+1, \ldots, m\}$. From the fact that $\bar{v} \in \mathcal{G}\left[\boldsymbol{A}_{11}\right]$, one can use this previous property to show that there exist nonnegative integers $u_{1}, \ldots, u_{k}$ such that the number of edges in $\pi$ with negative weight is precisely $\sum_{v=1}^{k} 2 u_{v}+2$. It follows that there are an even number of edges with negative weight in $\pi$, meaning $\pi$ is not a negative undirected cycle. This analysis holds for every undirected cycle in $\mathcal{G}[\boldsymbol{A}]$. We conclude that there does not exist a negative undirected cycle in $\mathcal{G}[\boldsymbol{A}]$, which implies that $\mathcal{G}[\boldsymbol{A}]$ is structurally balanced.

We have thus proved that there exists an undirected negative cycle in $\mathcal{G}[\boldsymbol{A}]$ if and only if there exists an undirected negative cycle in $\mathcal{G}[\boldsymbol{C}]$, which implies the structural balance or unbalance of $\mathcal{G}[\boldsymbol{A}]$ is the same as that of $\mathcal{G}\left[\boldsymbol{C}_{i}\right], \forall i \in \mathcal{I}$.

Part 2: Consider now the case when there are individuals with competing logical interdependencies. Suppose that there exist individuals $j, k$ such that $c_{p q, j}>0$ and $c_{p q, k}<0$ has negative sign (i.e. there are competing logical interdependencies in topic $p$ ). From Item 1) of Lemma 2, we know that the subgraph $\mathcal{G}\left[\boldsymbol{A}_{p p}\right]$ is strongly connected and all edges between nodes within $\mathcal{G}\left[\boldsymbol{A}_{p p}\right]$ have positive weight. From Item 2) and Item 3 ) of Lemma 2 , and because $w_{i i}>0$ for all $i$, we observe that $\mathcal{G}[\boldsymbol{A}]$ has an undirected cycle

$$
\begin{aligned}
& \left(v_{(q-1) n+j}, v_{(p-1) n+j}\right),\left(v_{(p-1) n+j}, v_{(p-1) n+z_{1}}\right), \ldots, \\
& \left(v_{(p-1) n+z_{r}}, v_{(p-1) n+k}\right),\left(v_{(p-1) n+k}, v_{(q-1) n+k}\right), \\
& \left(v_{(q-1) n+k}, v_{(q-1) n+z_{r}}\right), \ldots,\left(v_{(q-1) n+z_{1}}, v_{(q-1) n+j}\right)
\end{aligned}
$$

with $z_{1}, \ldots z_{r} \in \mathcal{I}$ and $r \geq 1$. The single negative edge is $\left(v_{(p-1) n+k}, v_{(q-1) n+k}\right)$, which means the undirected cycle is negative. It follows that $\mathcal{G}[\boldsymbol{A}]$ is structurally unbalanced.

\section{Proof of Theorem 2}

We first prove Statement 1). If there are no competing logical interdependencies and $\mathcal{G}\left[\boldsymbol{C}_{i}\right], \forall i \in \mathcal{I}$ are structurally balanced, then $\mathcal{G}[\boldsymbol{A}]$ is structurally balanced according to Lemma 3. According to [10, Theorem 1], for almost all initial conditions the system Eq. (8) converges to a nonzero modulus consensus, i.e. $\lim _{t \rightarrow \infty}\left|y_{p}^{i}(t)\right|=\left|y_{q}^{j}(t)\right| \neq 0$ for all $i, j \in \mathcal{I}$ and $p, q \in \mathcal{J}$. It remains to prove that $\lim _{t \rightarrow \infty} \boldsymbol{y}_{k}(t)=$ $\alpha_{k} \mathbf{1}_{n}, \forall k \in \mathcal{J}$.

For a structurally balanced $\mathcal{G}[\boldsymbol{A}]$, the nodes $v_{i} \in \mathcal{V}$ can be partitioned into two disjoint sets $\mathcal{V}^{+}$and $\mathcal{V}^{-}$, where every edge between nodes in the same set has positive weight, and every edge between nodes of $\mathcal{V}^{+}$and $\mathcal{V}^{-}$has negative weight. Item 1) of Lemma 2 implies that for any $k \in \mathcal{J}$, the nodes $v_{(k-1) n+1}, \ldots, v_{k n}$ all belong in either $\mathcal{V}^{+}$or $\mathcal{V}^{-}$. Recalling that the node $v_{(k-1) n+i}$ corresponds to the variable $y_{k}^{i}$, and from [10, Theorem 1], it follows that $\lim _{t \rightarrow \infty} y_{k}^{i}(t)=y_{k}^{j}(t)$ for all $i, j \in \mathcal{I}$, and thus $\lim _{t \rightarrow \infty} \boldsymbol{y}_{k}=\alpha_{k} \mathbf{1}_{n}$ for every $k \in \mathcal{J}$.

Statements 2) and 3) can be proved simultaneously. If there are no competing logical interdependencies, and $\mathcal{G}\left[\boldsymbol{C}_{i}\right], \forall i \in$ $\mathcal{I}$ are structurally unbalanced then according to Lemma 3 , $\mathcal{G}[\boldsymbol{A}]$ is structurally unbalanced. Similarly, if there are competing logical interdependencies, then according to Lemma 3, $\mathcal{G}[\boldsymbol{A}]$ is also structurally unbalanced. From [10, Theorem 2], there holds $\lim _{t \rightarrow \infty} \boldsymbol{y}(t)=\mathbf{0}_{n m}$ exponentially fast. This completes the proof of the theorem.

\section{Proof of Corollary 1}

Recall from Appendix B that the structural balance or unbalance property of any graph depends on the sign, and not the magnitude, of its edge weights. Since $\mathcal{G}\left[\boldsymbol{C}_{i}\right], \forall i \in \mathcal{I}$ are structurally balanced, we can consider $\mathcal{G}\left[\boldsymbol{C}_{1}\right]$ for convenience. For brevity, we also drop the subscript 1 and simply write $\mathcal{G}[\boldsymbol{C}]$. Partition the nodes $v_{1}, \ldots, v_{m}$ of $\mathcal{G}[\boldsymbol{C}]$ into two disjoint sets $\mathcal{V}[\boldsymbol{C}]^{+}$and $\mathcal{V}[\boldsymbol{C}]^{-}$such that every edge between nodes in the same set has positive weight, and every edge between nodes of different sets has negative weight.

Since $\mathcal{G}[\boldsymbol{A}]$ is structurally balanced, let us also partition the nodes $\tilde{v}_{k}$ of $\mathcal{G}[\boldsymbol{A}]$ into two disjoint sets $\mathcal{V}^{+}$and $\mathcal{V}^{-}$such that every edge between nodes in the same set has positive weight, and every edge between nodes of different sets has negative weight. We know from Lemma 2 Item 1) and Lemma 3 that the nodes $\tilde{v}_{(p-1) n+1}, \ldots, \tilde{v}_{p n}$ of $\mathcal{G}[\boldsymbol{A}]$ all belong in either $\mathcal{V}^{+}$ or $\mathcal{V}^{-}$. Recall from Item 2) and 3) of Lemma 2 that the weights 
of the edges from subgraph $\mathcal{G}\left[\boldsymbol{A}_{q q}\right]$ to subgraph $\mathcal{G}\left[\boldsymbol{A}_{p p}\right]$, with $p \neq q$ and $p, q \in \mathcal{J}$, have the same sign as the edges in $\mathcal{G}[\boldsymbol{C}]$ from $v_{q}$ to $v_{p}$. One can then use the analysis in [10] and Theorem 2, Statement 1), to verify that $\alpha_{p}=\alpha_{q}$ if $v_{p}$ and $v_{q}$ are either both in $\mathcal{V}[\boldsymbol{C}]^{+}$or both in $\mathcal{V}[\boldsymbol{C}]^{-}$. If, on the other hand, $v_{q} \in \mathcal{V}[\boldsymbol{C}]^{+}$and $v_{p} \in \mathcal{V}[\boldsymbol{C}]^{-}$, then $\alpha_{p}=-\alpha_{q}$.

\section{E. Proof of Theorem 3}

First, observe that if $\mathcal{J}_{j}=\{p\}$ is a singleton, then the block diagonal matrix $\overline{\boldsymbol{A}}_{p p}$ in Eq. (22) is in fact $\overline{\boldsymbol{A}}_{p p}=\boldsymbol{A}_{p p}=$ $\boldsymbol{\Gamma}_{p p} \boldsymbol{W}$, where $\boldsymbol{\Gamma}_{p q}, p, q \in \mathcal{J}$ is defined below Eq. (7). Since $0<c_{p p, i}<1$ for all $i \in \mathcal{I}$, and $\boldsymbol{W}$ is row-stochastic, we have that $\left\|\boldsymbol{A}_{p p}\right\|_{\infty}<1 \Rightarrow \rho\left(\boldsymbol{A}_{p p}\right)<1$. This implies that $\left(\boldsymbol{I}_{n}-\boldsymbol{A}_{p p}\right)^{-1}$ exists. Letting $\hat{\mathcal{J}}_{p}$ be the set of topics that topic $p$ logically depends upon, as defined in Eq. (11), the vector $\boldsymbol{y}_{p}(t)$ converges exponentially fast to

$$
\lim _{t \rightarrow \infty} \boldsymbol{y}_{p}(t) \triangleq \boldsymbol{y}_{p}^{*}=\left(\boldsymbol{I}_{n}-\boldsymbol{A}_{p p}\right)^{-1}\left(\sum_{j \in \hat{\mathcal{J}}_{p}} \boldsymbol{A}_{p j} \boldsymbol{y}_{j}^{*}\right) .
$$

We now focus on proving that $\boldsymbol{y}_{p}^{*}$ reaches a consensus state if and only if Eq. (13) holds for some $\kappa_{p} \in[-1,1]$. Let $\boldsymbol{R}_{p p}=$ $\boldsymbol{I}_{n}-\boldsymbol{A}_{p p}$, and because $\boldsymbol{W}$ is row-stochastic, one obtains that $\boldsymbol{A}_{p q} \mathbf{1}_{n}=\boldsymbol{\Gamma}_{p q} \mathbf{1}_{n}$ for any $q, p \in \mathcal{J}$ and $\boldsymbol{R}_{p p} \mathbf{1}_{n}=\left(\boldsymbol{I}_{n}-\right.$ $\left.\boldsymbol{\Gamma}_{p p}\right) \mathbf{1}_{n}$. We use this observation several times below.

Sufficiency: Suppose there exists a $\kappa_{p} \in[-1,1]$ satisfying Eq. (13). Since the theorem hypothesises that $\boldsymbol{y}_{q}^{*}=$ $\alpha_{q} \mathbf{1}_{n} \forall q \in \hat{\mathcal{J}}_{p}$, substituting Eq. (13) into Eq. (25) yields $\boldsymbol{y}_{p}^{*}=\boldsymbol{R}_{p p}^{-1}\left(\sum_{q \in \hat{\mathcal{J}}_{p}} \alpha_{q} \boldsymbol{\Gamma}_{p q}\right) \mathbf{1}_{n}=\kappa_{p} \mathbf{1}_{n}$. Also, $\alpha_{p}=\kappa_{p}$.

Necessity: Suppose in order to obtain a contradiction, that $\boldsymbol{y}_{p}^{*}=\alpha_{p} \mathbf{1}_{n}$ for some $\alpha_{p}$ and there does not exist a $\kappa_{p} \in$ $[-1,1]$ satisfying Eq. (13). Substituting $\boldsymbol{y}_{p}^{*}=\alpha_{p} \mathbf{1}_{n}$ into the left hand side of Eq. (25), we obtain

$$
\alpha_{p} \mathbf{1}_{n}=\boldsymbol{R}_{p p}^{-1}\left(\sum_{q \in \hat{\mathcal{J}}_{p}} \alpha_{q} \boldsymbol{\Gamma}_{p q}\right) \mathbf{1}_{n}
$$

Multiplying both sides by $\boldsymbol{R}_{p p}$ yields

$$
\alpha_{p}\left(\boldsymbol{I}_{n}-\boldsymbol{\Gamma}_{p p}\right) \mathbf{1}_{n}=\left(\sum_{q \in \hat{\mathcal{J}}_{p}} \alpha_{q} \boldsymbol{\Gamma}_{p q}\right) \mathbf{1}_{n} .
$$

However, Eq. (26) clearly contradicts the assumption made at the start of the proof of necessity: there does not exist a $\kappa_{p} \in[-1,1]$ satisfying Eq. (13) for all $i \in \mathcal{I}$.

\section{F. Proof of Corollary 2}

We prove each statement of Corollary 2 separately. First, note that $\left|\alpha_{q}\right| \leq 1$, which implies that the quantity on the right hand side of Eq. (13) is in $[-1,1]$ for every $i \in \mathcal{I}$.

Statement 1): First, observe that $\boldsymbol{I}_{n}-\boldsymbol{\Gamma}_{p p}=\left|\boldsymbol{\Gamma}_{p q}\right|$. For the proof of sufficiency, suppose that there are no competing logical interdependencies in topic $p$. Then, $\alpha_{q} c_{p q, i}$ has the same sign for every $i \in \mathcal{I}$ and since $\hat{\mathcal{J}}_{p}=\{q\}, \kappa_{p}=$ $\alpha_{q} \operatorname{sgn}\left(c_{p q, i}\right) \in[-1,1]$ satisfies Eq. (13), where the signum function $\operatorname{sgn}: \mathbb{R} \rightarrow\{-1,0,1\}$ satisfies $\operatorname{sgn}(x)=1$ if $x>0$, $\operatorname{sgn}(x)=0$ if $x=0$, and $\operatorname{sgn}(x)=-1$ if $x<0$.

For the proof of necessity, suppose that there are competing logical interdependencies in topic $p$, and suppose $c_{p q, 1}>0$ and $c_{p q, 2}<0$ (see below Eq. (5) on why we can make this assumption without loss of generality). Then, $\operatorname{sgn}\left(\alpha_{q} c_{p q, 1}\right)=$ $-\operatorname{sgn}\left(\alpha_{q} c_{p q, 2}\right)$, which implies that there does not exist a $\kappa_{p}$ such that Eq. (13).

Statement 2): The proof is trivial, since the right hand side of Eq. (13) is a zero matrix.

Statement 3): The sum constraint in Assumption 1 yields that $c_{p p, i}=c_{p p, j}=c_{p p}$ and $c_{p q_{k}, i}=c_{p q_{k}, j}=c_{p q_{k}}$ for all $k \in\{1, \ldots, r\}$ and $i, j \in \mathcal{I}$. This also implies that $\boldsymbol{\Gamma}_{p q_{k}}=c_{p q_{k}} \boldsymbol{I}_{n}$. Thus, Eq. (13) is equivalent to $\kappa_{p}=$ $\sum_{k=1}^{r} \alpha_{q_{k}} c_{p q_{k}} /\left(\sum_{k=1}^{r}\left|c_{p q_{k}}\right|\right)$. Since $\alpha_{q_{k}} \in[-1,1]$, there exists a $\kappa_{p} \in[-1,1]$ satisfying Eq. (13).

Statement 4): Let $\boldsymbol{z}_{k}=\left[c_{p q_{k}, 1}, \ldots, c_{p q_{k}, n}\right]^{\top}$, and $\Xi_{k}$ be the diagonal matrix with $i^{\text {th }}$ diagonal entry being $\operatorname{sgn}\left(c_{p q_{k}, i}\right)$. Then, Eq. (13) is equivalent to

$$
\kappa_{p} \sum_{k=1}^{r} \Xi_{k} \boldsymbol{z}_{k}=\sum_{k=1}^{r} \alpha_{q_{k}} \boldsymbol{z}_{k}
$$

since $1-c_{p p, i}=\sum_{q \neq p}\left|c_{p q, i}\right|$ for all $i$. Because we assumed that $\left|\alpha_{q_{u}}\right|=\left|\alpha_{q_{v}}\right|$ for all $u, v \in\{1, \ldots, r\}$, let $\bar{\alpha} \triangleq\left|\alpha_{q_{k}}\right|$.

In the case of (i), where $\operatorname{sgn}\left(c_{p q_{k}, i}\right)=\operatorname{sgn}\left(\alpha_{q_{k}}\right)$ for every $k \in\{1, \ldots, r\}$, it follows that $\alpha_{q_{k}} \boldsymbol{z}_{k}=\bar{\alpha} \Xi_{k} \boldsymbol{z}_{k}$. Rearranging Eq. (27) yields $\mathbf{0}_{n}=\sum_{k=1}^{r}\left(\kappa_{p}-\bar{\alpha}\right) \Xi_{k} \boldsymbol{z}_{k}$. Since $\bar{\alpha} \in[-1,1]$, choosing $\kappa_{p}=\bar{\alpha}$ ensures that Eq. (27) holds. The proof for case (ii) is the same, except that $\alpha_{q_{k}} \boldsymbol{z}_{k}=-\bar{\alpha} \Xi_{k} \boldsymbol{z}_{k}$ and one selects $\kappa_{p}=-\bar{\alpha}$ to satisfy Eq. (27).

\section{G. Proof of Theorem 4}

First, note that for any $p, q \in \mathcal{J}, \boldsymbol{A}_{p q} \mathbf{1}_{n}=\boldsymbol{\Gamma}_{p q} \mathbf{1}_{n}$, where $\boldsymbol{\Gamma}_{p q}$ has been defined below Eq. (7). For notational convenience, let $\mathcal{J}_{j}=\left\{j_{1}, \ldots, j_{z}\right\}$ with $z \geq 2$. In other words, we replace for brevity $\sum_{i=1}^{j} s_{i-1}+p$ in Eq. (10) with $j_{p}$, for $p=1, \ldots, z$. We proved in Theorem 1 that $\rho\left(\overline{\boldsymbol{A}}_{j j}\right)<1 \forall j \in \mathcal{S}$ which, combined with the assumption that $\boldsymbol{y}_{q}^{*}=\alpha_{q} \mathbf{1}_{n}, \alpha_{q} \in[-1,1]$ for all $q \in \tilde{\mathcal{J}}_{j}$, yields

$$
\left[\begin{array}{c}
\boldsymbol{y}_{j_{1}}^{*} \\
\vdots \\
\boldsymbol{y}_{j_{z}}^{*}
\end{array}\right]=\left(\boldsymbol{I}_{n z}-\overline{\boldsymbol{A}}_{j j}\right)^{-1}\left[\begin{array}{c}
\sum_{q \in \tilde{\mathcal{J}}_{j}} \alpha_{q} \boldsymbol{\Gamma}_{j_{1} q} \mathbf{1}_{n} \\
\vdots \\
\sum_{q \in \tilde{\mathcal{J}}_{j}} \alpha_{q} \boldsymbol{\Gamma}_{j_{z} q} \mathbf{1}_{n}
\end{array}\right]
$$

where $\boldsymbol{y}_{k}^{*} \triangleq \lim _{t \rightarrow \infty} \boldsymbol{y}_{k}(t)$ for $k \in \mathcal{J}_{j}$.

Sufficiency: Let $\Phi \in \mathbb{R}^{z \times z}$ be a diagonal matrix with $i^{\text {th }}$ diagonal entry $\phi_{k}$ and define

$$
\overline{\boldsymbol{C}}=\left[\begin{array}{ccc}
\boldsymbol{\Gamma}_{j_{1} j_{1}} & \ldots & \boldsymbol{\Gamma}_{j_{1} j_{z}} \\
\vdots & \ddots & \vdots \\
\boldsymbol{\Gamma}_{j_{z} j_{1}} & \ldots & \boldsymbol{\Gamma}_{j_{z} j_{z}}
\end{array}\right]
$$

Observe that

$$
\begin{aligned}
\overline{\boldsymbol{C}}\left(\boldsymbol{\Phi} \otimes \boldsymbol{I}_{n}\right)\left(\mathbf{1}_{z} \otimes \mathbf{1}_{n}\right) & =\overline{\boldsymbol{C}}(\boldsymbol{\Phi} \otimes \boldsymbol{W})\left(\mathbf{1}_{z} \otimes \mathbf{1}_{n}\right) \\
& =\overline{\boldsymbol{C}}\left(\boldsymbol{I}_{z} \otimes \boldsymbol{W}\right)\left(\boldsymbol{\Phi} \otimes \boldsymbol{I}_{n}\right)\left(\mathbf{1}_{z} \otimes \mathbf{1}_{n}\right) \\
& =\overline{\boldsymbol{A}}_{j j}\left(\boldsymbol{\Phi} \otimes \boldsymbol{I}_{n}\right)\left(\mathbf{1}_{z} \otimes \mathbf{1}_{n}\right)
\end{aligned}
$$

with the first equality obtained by recalling that $\boldsymbol{W} \mathbf{1}_{n}=\mathbf{1}_{n}$, and the last equality obtained by verifying from Eq. (23) that 
$\overline{\boldsymbol{A}}_{j j} \triangleq \overline{\boldsymbol{C}}\left(\boldsymbol{I}_{z} \otimes \boldsymbol{W}\right)$. If Eq. (15) holds, then by substituting the right-hand side of Eq. (15) into the left-hand side of Eq. (28), it follows that

$$
\begin{aligned}
{\left[\begin{array}{c}
\boldsymbol{y}_{j_{1}}^{*} \\
\vdots \\
\boldsymbol{y}_{j_{z}}^{*}
\end{array}\right] } & =\left(\boldsymbol{I}_{n z}-\overline{\boldsymbol{A}}_{j j}\right)^{-1}\left(\boldsymbol{I}_{n z}-\overline{\boldsymbol{C}}\right)\left(\boldsymbol{\Phi} \otimes \boldsymbol{I}_{n}\right) \mathbf{1}_{n z} \\
& =\left(\boldsymbol{\Phi} \otimes \boldsymbol{I}_{n}\right) \mathbf{1}_{n z},
\end{aligned}
$$

with the first equality obtained verifying that $\bar{C}\left(\phi \otimes \mathbf{1}_{n}\right)=$ $\overline{\boldsymbol{C}}\left(\boldsymbol{\Phi} \otimes \boldsymbol{I}_{n}\right)\left(\mathbf{1}_{z} \otimes \mathbf{1}_{n}\right)$, and the last equality obtained from Eq. (30). It follows that $\boldsymbol{y}_{k}^{*}=\phi_{k} \mathbf{1}_{n}$ for every $k \in \mathcal{J}_{j}$.

Necessity: To obtain a contradiction, suppose that $\nexists \phi$ with each entry having modulus less than or equal to 1 such that Eq. (15) holds, and (ii) there holds $\boldsymbol{y}_{j_{k}}^{*}=\alpha_{j_{k}} \mathbf{1}_{n}$ for some $\alpha_{j_{k}} \in[-1,1]$, for all $j_{k} \in \mathcal{J}_{j}$. Note that $\alpha_{k_{p}} \in[-1,1], k_{p} \in$ $\tilde{\mathcal{J}}_{j}$ is a consequence of the invariance property of Eq. (8) (see Theorem 1). Eq. (28) yields

$$
\left[\begin{array}{c}
\alpha_{j_{1}} \mathbf{1}_{n} \\
\vdots \\
\alpha_{j_{z}} \mathbf{1}_{n}
\end{array}\right]=\left(\boldsymbol{I}_{n z}-\overline{\boldsymbol{A}}_{j j}\right)^{-1}\left[\begin{array}{c}
\sum_{q \in \tilde{\mathcal{J}}_{j}} \alpha_{q} \boldsymbol{\Gamma}_{j_{1} q} \mathbf{1}_{n} \\
\vdots \\
\sum_{q \in \tilde{\mathcal{J}}_{j}} \alpha_{q} \boldsymbol{\Gamma}_{j_{z} q} \mathbf{1}_{n}
\end{array}\right]
$$

Let $\overline{\boldsymbol{\alpha}}$ be a diagonal matrix with $i^{\text {th }}$ diagonal entry $\alpha_{j_{k}}$. Recalling that $\overline{\boldsymbol{A}}_{j j} \triangleq \overline{\boldsymbol{C}}\left(\boldsymbol{I}_{z} \otimes \boldsymbol{W}\right)$, multiply both sides of Eq. (32) by $\boldsymbol{I}_{n z}-\overline{\boldsymbol{A}}_{j j}$. Simplifying using calculations similar to those appearing in Eq. (30) but with $\overline{\boldsymbol{\alpha}}$ replacing $\boldsymbol{\Phi}$ yields

$$
\left(\boldsymbol{I}_{n z}-\overline{\boldsymbol{C}}\right)\left(\overline{\boldsymbol{\alpha}} \otimes \mathbf{1}_{n}\right)=\left[\begin{array}{c}
\sum_{q \in \tilde{\mathcal{J}}_{j}} \alpha_{q} \boldsymbol{\Gamma}_{j_{1} q} \\
\vdots \\
\sum_{q \in \tilde{\mathcal{J}}_{j}} \alpha_{q} \boldsymbol{\Gamma}_{j_{z} q}
\end{array}\right] \mathbf{1}_{n z}
$$

However, Eq. (33) contradicts the assumption made at the start of this (necessity) part of the proof: there does not exist a vector $\phi$ such that Eq. (15). One can prove that each entry of $\overline{\boldsymbol{\alpha}}$ in Eq. (33) is less than or equal to 1 by exploiting the sum constraint on the $c_{p q, i}$ in Assumption 1, and the fact that $\alpha_{q} \in[-1,1]$ for all $q \in \mathcal{J}_{j}$. Using calculations similar to those at the end of Appendix A, one can alsso show that $\rho(\overline{\boldsymbol{C}})<1$, which establishes the invertibility of $\boldsymbol{I}_{n z}-\overline{\boldsymbol{C}}$.

\section{H. Proof of Corollary 3}

We prove each item separately.

Item 1: This result can be immediately obtained by checking Eq. (15) with $\alpha_{q}=0$ for all $q \in \tilde{\mathcal{J}}_{j}$.

Item 2: First, note that $\boldsymbol{C}_{i}$ is of the form in Eq. (9), which implies that $c_{k a, i}=0$ for all $k \in \mathcal{J}_{j}, a>\max \mathcal{J}_{j}$, and $i \in \mathcal{I}$. Let $\overline{\boldsymbol{A}}_{j j}$ be defined as in Eq. (23). Similar to the proof of Theorem 4 , let $\mathcal{J}_{j}=\left\{j_{1}, \ldots, j_{z}\right\}$ with $z \geq 2$. Supposing that $c_{j_{k} p, g}=c_{j_{k} p, h}=c_{j_{k} p}$ for $j_{k} \in \mathcal{J}_{j}$ and $p \in \mathcal{J}$, define

$$
\hat{\boldsymbol{C}}=\left[\begin{array}{ccc}
c_{j_{1} j_{1}} & \ldots & c_{j_{1} j_{z}} \\
\vdots & \ddots & \vdots \\
c_{j_{z} j_{1}} & \ldots & c_{j_{z} j_{z}}
\end{array}\right]
$$

Then, $\boldsymbol{I}-\overline{\boldsymbol{A}}_{j j}=\boldsymbol{I}_{n z}-\hat{\boldsymbol{C}} \otimes \boldsymbol{W}$. Since $\rho\left(\overline{\boldsymbol{A}}_{j j}\right)<1$, we obtain from the Neumann series that $\left(\boldsymbol{I}-\overline{\boldsymbol{A}}_{j j}\right)^{-1}=\sum_{t=0}^{\infty} \overline{\boldsymbol{A}}_{j j}{ }^{t}=$ $\sum_{t=0}^{\infty} \hat{\boldsymbol{C}}^{t} \otimes \boldsymbol{W}^{t}$. Assumption 1 and the fact that $\tilde{\mathcal{J}}_{j} \neq \emptyset$ implies that $|\hat{\boldsymbol{C}}|$ is row-substochastic. Using calculations similar to those at the end of Appendix A, one can show that $\rho(\hat{\boldsymbol{C}})<1$, which establishes the existence of $\sum_{t=0}^{\infty} \hat{\boldsymbol{C}}^{t}$.

Define for $k \in\{1, \ldots z\}, \quad \tilde{\alpha}_{j_{k}}=\sum_{q \in \tilde{\mathcal{J}}_{j}} \alpha_{q} c_{k_{p} q}$, and observe that

$$
\left[\begin{array}{c}
\sum_{q \in \tilde{\mathcal{J}}_{j}} \alpha_{q} \boldsymbol{\Gamma}_{j_{1} q} \mathbf{1}_{n} \\
\vdots \\
\sum_{q \in \tilde{\mathcal{J}}_{j}} \alpha_{q} \boldsymbol{\Gamma}_{j_{z} q} \mathbf{1}_{n}
\end{array}\right]=\tilde{\boldsymbol{\alpha}} \otimes \mathbf{1}_{n}
$$

where $\tilde{\boldsymbol{\alpha}}=\left[\tilde{\alpha}_{j_{1}}, \ldots, \tilde{\alpha}_{j_{z}}\right]^{\top}$. We obtain from Eq. (28) that

$$
\left[\begin{array}{c}
\boldsymbol{y}_{j_{1}}^{*} \\
\vdots \\
\boldsymbol{y}_{j_{z}}^{*}
\end{array}\right]=\left(\sum_{t=0}^{\infty} \hat{\boldsymbol{C}}^{t} \otimes \boldsymbol{W}^{t}\right) \tilde{\boldsymbol{\alpha}} \otimes \mathbf{1}_{n}=\left(\sum_{t=0}^{\infty} \hat{\boldsymbol{C}}^{t} \tilde{\boldsymbol{\alpha}}\right) \otimes \mathbf{1}_{n}
$$

since $\boldsymbol{W}^{t}$ is a row-stochastic matrix for any $t \in \mathbb{N}$. The right hand side of Eq. (36) is equal to $\boldsymbol{u} \otimes \mathbf{1}_{n}$ for $\boldsymbol{u}=\sum_{t=0}^{\infty} \hat{\boldsymbol{C}}^{t} \tilde{\boldsymbol{\alpha}} \in$ $\mathbb{R}^{z}$, which implies that for every $j_{k} \in \mathcal{J}_{j}$, we have $\boldsymbol{y}_{j_{k}}^{*}=$ $\alpha_{j_{k}} \mathbf{1}_{n}$ for some $\alpha_{j_{k}} \in[-1,1]$.

\section{ACKNOWLEDGEMENT}

We thank the Editor and anonymous reviewers for their invaluable comments which improved this paper.

\section{REFERENCES}

[1] A. V. Proskurnikov and R. Tempo, "A tutorial on modeling and analysis of dynamic social networks. Part I," Annual Reviews in Control, vol. 43, pp. 65-79, 2017.

[2] A. Flache, M. Mäs, T. Feliciani, E. Chattoe-Brown, G. Deffuant, S. Huet, and J. Lorenz, "Models of Social Influence: Towards the Next Frontiers," Journal of Artificial Societies \& Social Simulation, vol. 20, no. 4, 2017.

[3] J. R. P. French Jr, "A Formal Theory of Social Power," Psychological Review, vol. 63, no. 3, pp. 181-194, 1956.

[4] F. Harary, Studies in social power. Ann Arbor, University of Michigan Press, 1959, ch. A criterion for unanimity in French's theory of social power, pp. 198-182.

[5] M. H. DeGroot, "Reaching a Consensus," Journal of the American Statistical Association, vol. 69, no. 345, pp. 118-121, 1974.

[6] R. Hegselmann and U. Krause, "Opinion dynamics and bounded confidence models, analysis, and simulation," Journal of Artificial Societies and Social Simulation, vol. 5, no. 3, 2002.

[7] V. D. Blondel, J. M. Hendrickx, and J. N. Tsitsiklis, "On Krause's MultiAgent Consensus Model with State-Dependent Connectivity," IEEE Transactions on Automatic Control, vol. 54, no. 11, pp. 2586-2597, 2009.

[8] W. Su, G. Chen, and Y. Hong, "Noise leads to quasi-consensus of hegselmann-krause opinion dynamics," Automatica, vol. 85, pp. 448 454, 2017.

[9] C. Altafini, "Consensus Problems on Networks with Antagonistic Interactions," IEEE Transactions on Automatic Control, vol. 58, no. 4, pp. 935-946, 2013.

[10] J. Liu, X. Chen, T. Başar, and M.-A. Belabbas, "Exponential Convergence of the Discrete- and Continuous-Time Altafini Models," IEEE Transaction on Automatic Control, vol. 62, no. 12, pp. 6168-6182, 2017.

[11] A. Proskurnikov, A. Matveev, and M. Cao, "Opinion dynamics in social networks with hostile camps: Consensus vs. polarization," IEEE Transaction on Automatic Control, vol. 61, no. 6, pp. 1524-1536, 2016.

[12] W. Xia, M. Cao, and K. Johansson, "Structural Balance and Opinion Separation in Trust-Mistrust Social Networks," IEEE Transactions on Control of Network Systems, vol. 3, no. 1, pp. 46-56, 2016. 
[13] N. E. Friedkin and E. C. Johnsen, "Social Influence and Opinions," Journal of Mathematical Sociology, vol. 15, no. 3-4, pp. 193-206, 1990.

[14] N. E. Friedkin and F. Bullo, "How truth wins in opinion dynamics along issue sequences," Proceedings of the National Academy of Sciences, vol. 114, no. 43, pp. 11380-11385, 2017.

[15] J. Becker, D. Brackbill, and D. Centola, "Network dynamics of social influence in the wisdom of crowds," Proceedings of the National Academy of Sciences, vol. 114, no. 26, pp. E5070-E5076, 2017.

[16] N. E. Friedkin and E. C. Johnsen, Social Influence Network Theory: A Sociological Examination of Small Group Dynamics. Cambridge University Press, 2011, vol. 33.

[17] A. V. Proskurnikov and R. Tempo, "A tutorial on modeling and analysis of dynamic social networks. Part II," Annual Reviews in Control, vol. 45, pp. 166-190, 2018.

[18] S. E. Parsegov, A. V. Proskurnikov, R. Tempo, and N. E. Friedkin, "Novel Multidimensional Models of Opinion Dynamics in Social Networks," IEEE Transactions on Automatic Control, vol. 62, no. 5, pp. 2270-2285, 2017.

[19] N. E. Friedkin, A. V. Proskurnikov, R. Tempo, and S. E. Parsegov, "Network science on belief system dynamics under logic constraints," Science, vol. 354, no. 6310, pp. 321-326, 2016.

[20] P. E. Converse, "The nature of belief systems in mass publics," Ideology and Discontent, pp. 206-61, 1964

[21] A. Nedić, A. Olshevsky, and C. A. Uribe, "Graph-theoretic analysis of belief system dynamics under logic constraints," Scientific Reports, vol. 9, no. 1, p. 8843, 2019 .

[22] M. Ye, J. Liu, and B. D. O. Anderson, "On the Effects of Heterogeneous Logical Interdependencies in Multi-Dimensional Opinion Dynamics Models," in Proceedings of the IEEE 57th Annual Conference on Decision and Control, Miami, USA, 2018, pp. 4372-4377.

[23] M. Mäs, A. Flache, and J. A. Kitts, "Cultural Integration and Differentiation in Groups and Organizations," in Perspectives on Culture and Agent-based Simulations. Springer, 2014, pp. 71-90.

[24] P. Duggins, "A Psychologically-Motivated Model of Opinion Change with Applications to American Politics," Journal of Artificial Societies and Social Simulation, vol. 20, no. 1, pp. 1-13, 2017.

[25] V. Amelkin, F. Bullo, and A. K. Singh, "Polar opinion dynamics in socia networks," IEEE Transactions on Automatic Control, vol. 62, no. 11, pp. 5650-5665, 2017.

[26] F. Bullo, J. Cortes, and S. Martinez, Distributed Control of Robotic Networks. Princeton University Press, 2009.

[27] D. Cartwright and F. Harary, "Structural Balance: A Generalization of Heider's Theory," Psychological review, vol. 63, no. 5, p. 277, 1956.

[28] D. E. Cartwright and A. E. Zander, Group Dynamics Research and Theory. Tavistock Publications: London, 1953

[29] M. Ye, J. Liu, L. Wang, B. D. O. Anderson, and M. Cao, "Consensus and Disagreement of Heterogeneous Belief Systems in Influence Networks," 2018, submitted journal paper. [Online]. Available: https://arxiv.org/abs/1812.05138

[30] P. Frasca, S. Tarbouriech, and L. Zaccarian, "Hybrid models of opinion dynamics with opinion-dependent connectivity," Automatica, vol. 100, pp. 153-161, 2019.

[31] M. H. McAndrew, "On the Product of Directed Graphs," Proceedings of the American Mathematical Society, vol. 14, no. 4, pp. 600-606, 1963.

[32] R. S. Varga, Matrix Iterative Analysis. Springer Science \& Business Media, 2009, vol. 27.

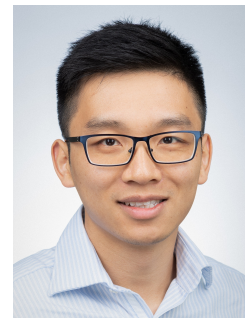

Mengbin Ye (S'13-M'18) was born in Guangzhou, China. He received the B.E. degree (with Firs Class Honours) in mechanical engineering from the University of Auckland, Auckland, New Zealand in 2013, and the Ph.D. degree in engineering at the Australian National University (ANU), Canberra, Australia in 2018. He is currently a postdoctoral researcher with the Faculty of Science and Engineering, University of Groningen, Netherlands.

He has been awarded the 2018 J.G. Crawford Prize (Interdisciplinary), ANU's premier award recognising graduate research excellence. He has also received the 2018 Springer PhD Thesis Prize, and was Highly Commended in the Best Student Paper Award at the 2016 Australian Control Conference. His current research interests include opinion dynamics and decision making in complex social networks, epidemic modelling, coordination of multi-agent systems, and localisation using bearing measurements.
Ji Liu (S'09-M'13) received the B.S. degree in information engineering from Shanghai Jiao Tong University, Shanghai, China, in 2006, and the Ph.D degree in electrical engineering from Yale University, New Haven, CT, USA, in 2013. He is currently an Assistant Professor in the Department of Electrical and Computer Engineering at Stony Brook University, Stony Brook, NY, USA. His curren research interests include distributed control and optimization, distributed machine learning, multiagent systems, social networks, epidemic networks, and cyber-physical systems.

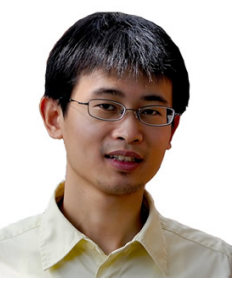

Lili Wang received the B.E. and M.S. degrees from Zhejiang University, Zhejiang, China, in 2011 and 2014, respectively. She is currently a Ph.D. student majored in electrical engineering in the School of Engineering \& Applied Science, Yale University, USA. Her research is on the topic of cooperative multi-agent systems and distributed observer.

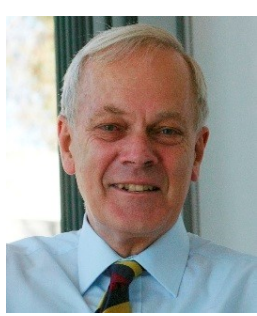

Brian D.O. Anderson (M'66-SM'74-F'75-LF'07) was born in Sydney, Australia. He received the B.Sc. degree in pure mathematics in 1962, and B.E. in electrical engineering in 1964, from the Sydney University, Sydney, Australia, and the Ph.D. degree in electrical engineering from Stanford University, Stanford, CA, USA, in 1966.

$\mathrm{He}$ is an Emeritus Professor at the Australian National University, and a Distinguished Researcher in Data61-CSIRO (previously NICTA) and a Distinguished Professor at Hangzhou Dianzi University. His awards include the IEEE Control Systems Award of 1997, the 2001 IEEE James H Mulligan, Jr Education Medal, and the Bode Prize of the IEEE Control System Society in 1992, as well as several IEEE and other best paper prizes. He is a Fellow of the Australian Academy of Science, the Australian Academy of Technological Sciences and Engineering, the Royal Society, and a foreign member of the US National Academy of Engineering. He holds honorary doctorates from a number of universities, including Université Catholique de Louvain, Belgium, and ETH, Zürich. He is a past president of the International Federation of Automatic Control and the Australian Academy of Science. His current research interests are in distributed control, sensor networks and econometric modelling.

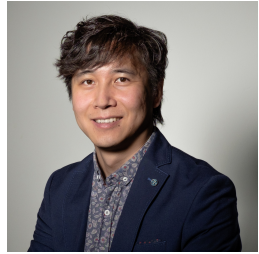

Ming Cao is currently Professor of systems and control with the Engineering and Technology Institute (ENTEG) at the University of Groningen, the Netherlands, where he started as a tenure-track Assistant Professor in 2008. He received the Bachelor degree in 1999 and the Master degree in 2002 from Tsinghua University, Beijing, China, and the $\mathrm{Ph} . \mathrm{D}$. degree in 2007 from Yale University, New Haven, CT, USA, all in Electrical Engineering. From September 2007 to August 2008, he was a Postdoctoral Research Associate with the Department of Mechanical and Aerospace Engineering at Princeton University, Princeton, NJ, USA. He worked as a research intern during the summer of 2006 with the Mathematical Sciences Department at the IBM T. J. Watson Research Center, NY, USA.

$\mathrm{He}$ is the 2017 and inaugural recipient of the Manfred Thoma medal from the International Federation of Automatic Control (IFAC) and the 2016 recipient of the European Control Award sponsored by the European Contro Association (EUCA). He is an Associate Editor for IEEE Transactions on Automatic Control, IEEE Transactions on Circuits and Systems and Systems and Control Letters, and for the Conference Editorial Board of the IEEE Control Systems Society. He is also a member of the IFAC Technical Committee on Networked Systems. His research interests include autonomous agents and multi-agent systems, mobile sensor networks and complex networks. 